\title{
AIP1 functions as an endogenous inhibitor of VEGFR2-mediated signaling and inflammatory angiogenesis in mice
}

\author{
Haifeng Zhang, ${ }^{1}$ Yun He, ${ }^{1}$ Shengchuan Dai, ${ }^{1}$ Zhe Xu, ${ }^{2}$ Yan Luo, ${ }^{2}$ Ting Wan, ${ }^{2}$ Dianhong Luo, ${ }^{1}$ \\ Dennis Jones, ${ }^{1}$ Shibo Tang, ${ }^{2}$ Hong Chen, ${ }^{1}$ William C. Sessa, ${ }^{1}$ and Wang Min ${ }^{1}$
}

\begin{abstract}
${ }^{1}$ Interdepartmental Program in Vascular Biology and Therapeutics, Department of Pathology, Yale University School of Medicine, New Haven, Connecticut, USA. ${ }^{2}$ Zhongshan Ophthalmic Center, Sun Yat-Sen University, Guangzhou, People's Republic of China.
\end{abstract}

\begin{abstract}
ASK1-interacting protein-1 (AIP1), a recently identified member of the Ras GTPase-activating protein family, is highly expressed in vascular ECs and regulates EC apoptosis in vitro. However, its function in vivo has not been established. To study this, we generated AIP1-deficient mice (KO mice). Although these mice showed no obvious defects in vascular development, they exhibited dramatically enhanced angiogenesis in 2 models of inflammatory angiogenesis. In one of these models, the enhanced angiogenesis observed in the $\mathrm{KO}$ mice was associated with increased VEGF-VEGFR2 signaling. Consistent with this, VEGF-induced ear, cornea, and retina neovascularization were greatly augmented in $\mathrm{KO}$ mice and the enhanced retinal angiogenesis was markedly diminished by overexpression of AIP1. In vitro, VEGF-induced EC migration was inhibited by AIP1 overexpression, whereas it was augmented by both AIP1 knockout and knockdown, with the enhanced EC migration caused by AIP1 knockdown being associated with increased VEGFR2 signaling. We present mechanistic data that suggest AIP1 is recruited to the VEGFR2-PI3K complex, binding to both VEGFR2 and PI3K p85, at a late phase of the VEGF response, and that this leads to inhibition of VEGFR2 signaling. Taken together, our data demonstrate that AIP1 functions as an endogenous inhibitor in VEGFR2-mediated adaptive angiogenesis in mice.
\end{abstract}

\section{Introduction}

Angiogenesis, the process of new blood vessel formation, is involved in many physiological and pathological settings such as ischemia, diabetes, atherosclerosis, and cancer (1). The VEGFs and their receptors have been shown to be critical in regulating vessel formation in both normal physiological and pathological processes (2). VEGF primarily utilizes its receptor VEGFR2 (also known as Flk-1 or KDR) to induce angiogenic responses by activating a variety of signaling cascades, including activation of PI3K-Akt, PLC- $\gamma-P K C$, and MAPK (3). Given the critical role of VEGFR2 signaling in angiogenesis, regulation of VEGFR2 activity/activation may represent an important mechanism for the control of angiogenesis. Many secreted antiangiogenic factors, including vasohibin, function, at least in part, by modulating VEGF bioactivity or the binding to VEGFR2 (4). In contrast, proangiogenic factors can positively regulate VEGF-VEGFR2 signaling. Sphingosine-1 phosphate via its receptor S1P1, placental growth factor via its receptor VEGFR1, and laminar flow via Src can transactivate VEGFR2 (5-8). We have also shown that proinflammatory cytokine TNF via its receptor TNFR2 transactivates VEGFR2 $(9,10)$. VEGFR2 activity is also regulated by direct interactions with other proteins, including co-receptor neuropilins (11), adhesion molecule vascular endothelial-cadherin (VE-cadherin) (12), and integrins (13). For example, VE-cadherin is in complex with VEGFR2 and is critical for VEGF-induced survival (PI3K-Akt) signaling (12).

Nonstandard abbreviations used: AIP1, ASK1-interacting protein-1; AIP1-F, fulllength AIP1; AIP1-N, AIP1 containing the N-terminal; ASK1, apoptosis signal-regulating kinase 1; GAP, GTPase-activating protein; GST, glutathione-S-transferase; $\mathrm{PH}$, pleckstrin homology; PR, proline-rich region; SH3, Src homology region 3; VE-cadherin, vascular endothelial-cadherin.

Conflict of interest: The authors have declared that no conflict of interest exists. Citation for this article: J. Clin. Invest. doi:10.1172/JCI36168.
VE-cadherin-VEGFR2 also regulates EC permeability $(14,15)$. Interestingly, a complex of VEGFR2, VE-cadherin, and PECAM-1 (CD31) has been shown to be a mechanosensor that functions upstream of integrin signaling and transduces shear stress-mediated angiogenesis and vascular remodeling (16). However, negative regulations of VEGFR2 by protein-protein interactions are less understood, and an endogenous inhibitor that directly binds to and modulates VEGFR2 activity has not been identified.

Apoptosis signal-regulating kinase 1-interacting (ASK1-interacting) protein-1 (AIP1), also known as DAB2-interacting protein (DAB2IP), a recently identified, novel member of the Ras GTPase-activating protein family, has been implicated in cell growth inhibition and cell apoptosis $(17,18)$. AIP1, via its Ras GTPase-activating protein (Ras-GAP) activity, inhibits Ras-mediated cell survival signaling, causing cell growth inhibition (19). On the other hand, AIP1 functions as a positive regulator in cell apoptosis by mediating activation of the apoptotic kinase apoptosis signal-regulating kinase 1 (ASK1) (18). Consistent with its role as an inhibitor of cell survival and growth, AIP1 expression is often downregulated in various human cancers $(17,20,21)$. Promoter analyses indicate that an epigenetic mechanism regulates AIP1 transcription (17). Recently, it has been reported that genetic variation in AIP1 gene may predict the risk of aggressive prostate cancer based on single nucleotide polymorphism (22, 23). Moreover, human AIP1 (alias for AFQ34) has been identified as a novel fusion partner from an acute myeloid leukemia patient with a $\mathrm{t}(9 ; 11)(\mathrm{q} 34 ; \mathrm{q} 23)$ chromosomal translocation; the intron 9 of the MLL gene is translocated into the exon 2 of AIP1 and causes the disruption of the pleckstrin homology $(\mathrm{PH})$ domain in the AIP1 protein and possible disruption of its functions in cell growth inhibition and apoptosis (24). These data suggest that AIP1 may function as a tumor suppressor gene. 
A

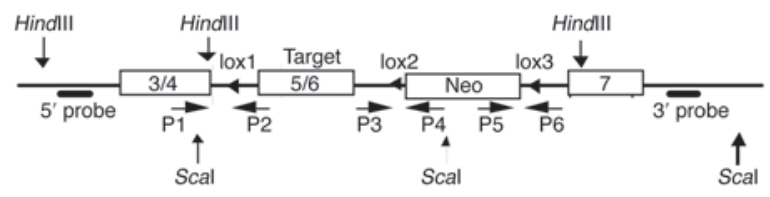

B Southern using 5' probe

ES clone no. $42 \quad 51 \quad 52 \quad 53$

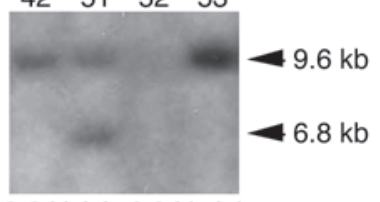

$9.6 \mathrm{~kb}(-) ; 6.8 \mathrm{~kb}(+)$
C

Southern using $3^{\prime}$ probe
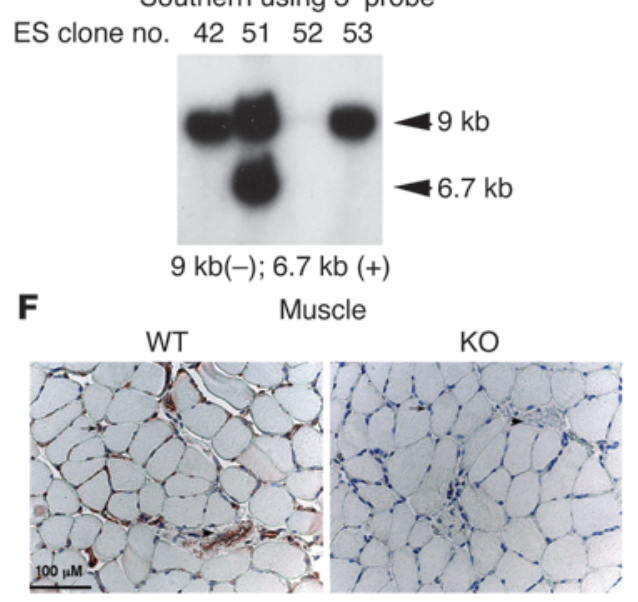

D

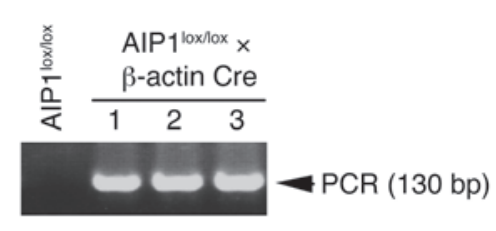

E

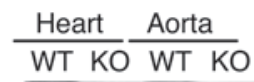

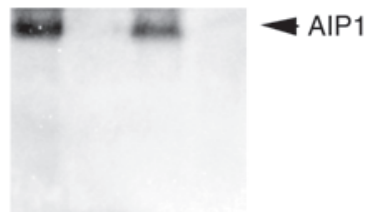

2-3-Tubulin

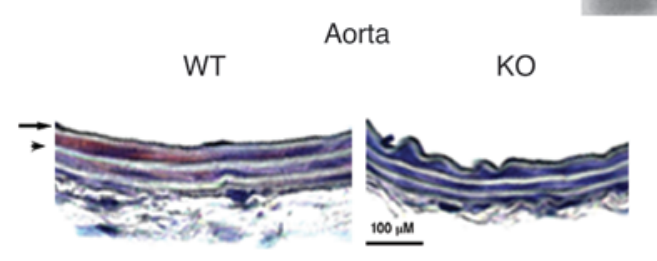

Figure 1

Generation of AIP1-flox and knockout mice. (A) Schematic diagram for strategy to generate AIP1-flox mice. The targeting vector contains exons $3 / 4$ at $5^{\prime}$ arm, exons 5/6 in the targeting region, and exon 7 at $3^{\prime}$ arm. PCR primers P1-P6 are shown. The 5' probe with Hindlll digest, and $3^{\prime}$ probe with Scal digest are indicated. (B and C) Screening of ES clones. Genomic DNA from positive clones was extracted and used for Southern blot, using 5' probe after HindIII digest (B) and 3' probe after Scal digest (C). Clone 51 was positive and used for blastocyst injection to generate chimera mice. (D) Generation of $A I P 1^{+/ l o x}$ and $A I P 1^{+-}$mice. The chimeras were mated with C57BL/6 for germline transmission to obtain AIP1+/lox. AIP1+/lox mice were further mated with $\beta$-actin Cre mice to delete both the targeting region (exons $5 / 6)$ and the Neo gene $\left(A I P 1^{+/-}\right.$mice). KO mice were obtained by mating between the heterozygous $\left(A I P 1^{+-}\right)$male and female. (E and F) AIP1 deletion in tissues. Muscle and aorta from WT and $\mathrm{KO}$ mice were homogenized and AIP1 expression was detected by Western blot with anti-AIP1 antibody (E). AIP1 expression in paraffin section of muscle and aorta was determined by immunohistochemistry with anti-AIP1 antibody (F). For muscle tissue, arrows indicate capillaries and the arrowhead indicates a large vessel. For aorta tissue, the arrow indicates endothelium and the arrowhead indicates smooth muscle layer.

However, the in vivo function of AIP1 has not been established. In order to define the in vivo function of AIP1, we created mice that were genetically deficient of the AIP1 gene. AIP1 is highly expressed in vascular ECs. However, AIP1-deficient mice (KO mice) show normal vascular development. We determined the function of AIP1 using several models of inflammatory angiogenesis. Our studies suggest that AIP1 functions as an endogenous inhibitor of adaptive angiogenesis, in part, by binding to the VEGFR2-PI3K complex and inhibiting the VEGFR2-dependent angiogenic signaling.

\section{Results}

Generation of KO mice. To explore the role of AIP1 in vivo, we created AIP1-knockout mice (KO mice) using homologous recombination (see Figure 1, A-D, and Methods for details). KO mice were backcrossed with C57BL/ 6 mice for 6 generations and they were viable and fertile. All experiments were performed with littermates $A I P 1^{+/+}(\mathrm{WT})$ and $A I P 1^{-/-}(\mathrm{KO})$ mice. AIP1 protein expression was detected by Western blot with anti-AIP1 antibodies recognizing either the $\mathrm{N}$-terminal $\mathrm{PH}$ domain or the $\mathrm{C}$-terminal end of AIP1. AIP1 protein in various tissues was detected by Western blot but not in KO tissues (Figure 1E for skeletal muscle and aorta tissues with anti-N-terminal AIP1 antibody).
AIP1 protein in various tissues was further determined by immunohistochemistry. Consistent with previous findings that AIP1 is highly expressed in vascular ECs (18), AIP1 protein was detected in vascular endothelium of muscle and aorta (Figure 1F). AIP1 expression was also detected in smooth muscle cells of aorta, epithelial cells of lung, and neurons of brain. However, $\mathrm{KO}$ mice showed no AIP1 expression in these tissues (Figure 1F and Supplemental Figure 1, A and B, respectively; supplemental material available online with this article; doi:10.1172/ JCI36168DS1). We also examined AIP1 expression during embryogenesis. AIP1 expression was detected in vasculature of WT but not KO embryos. However, KO mice show normal vascular development (Supplemental Figure 2). KO adult mice also show gross growth and breeding.

KO mice demonstrated enhanced inflammatory angiogenesis in vivo. Given that AIP1 is highly expressed in vascular endothelium, we decided to determine the function of AIP1 in adaptive angiogenesis. To this end, WT and KO mice were subjected to commonly used angiogenesis models, including hind limb ischemia and foreign body response to implanted sponge. WT and KO male mice were subjected to femoral artery ligation and various analyses at different time points as described recently (10). Blood flow was 


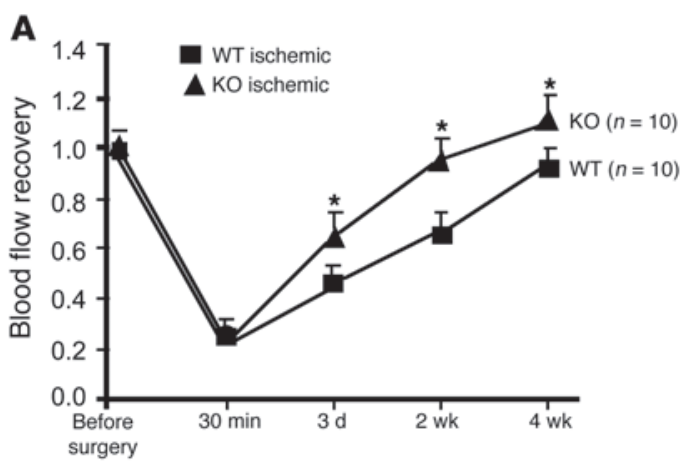

D

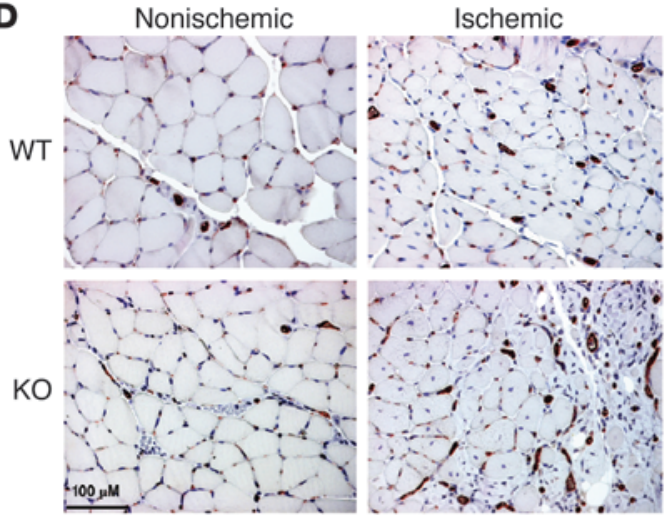

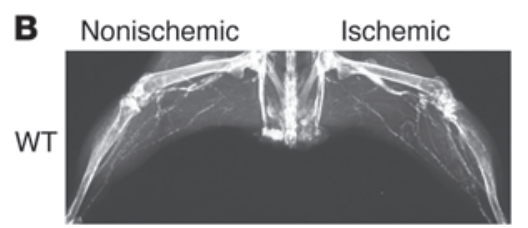

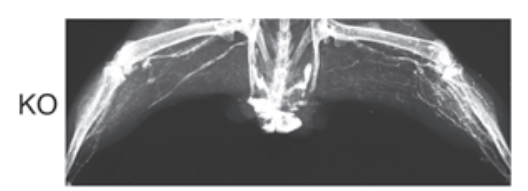

E

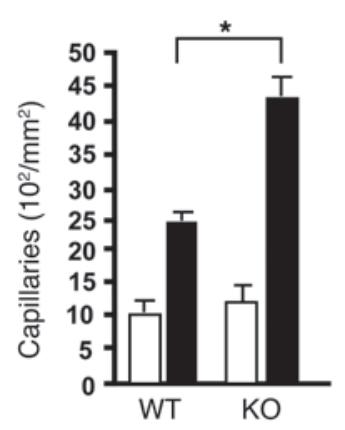

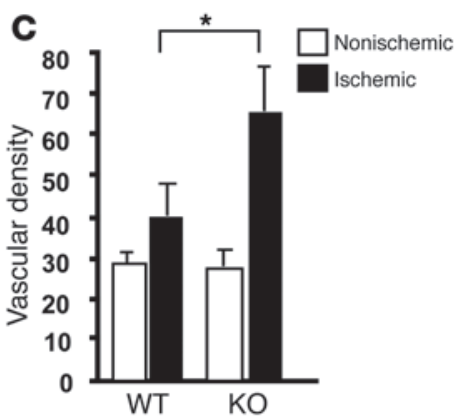

$\mathbf{F}$

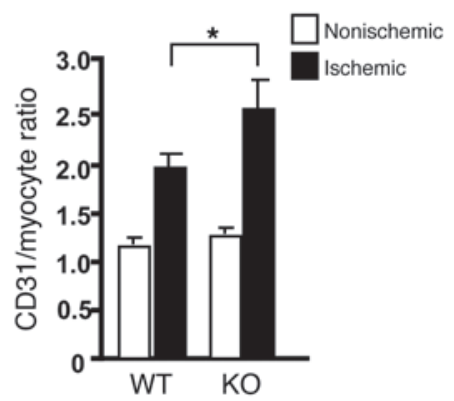

Figure 2

$\mathrm{KO}$ mice showed enhanced arteriogenesis and angiogenesis in vivo. (A) KO mice showed augmented recovery of limb perfusion compared with WT mice. Blood flow of ischemic and nonischemic limbs were measured in gastronomic muscle at before surgery and 30 minutes, 3 days, 2 weeks, and 4 weeks after surgery. Data (mean \pm SEM) are presented as the ratio of perfusion unit from nonischemic leg to ischemic leg. ${ }^{*} P<0.05$. (B and C) Angiographic evidence for enhanced arteriogenesis in KO mice. Arterial phase angiograms from WT and KO mice after 4 weeks of ischemia (B) were determined by quantitative angiography (C). Data are mean values from 2 angiograms. ${ }^{*} P<0.05$. (D-F) Enhanced angiogenesis in KO mice. Capillary density and pericyte recruitment were immunostained with CD31 (an EC marker). Representative sections from nonischemic and ischemic groups of WT and KO mice on day 28 after ischemia are shown in $\mathbf{D}$. Quantification of capillary density (number/mm $\mathrm{mm}^{2} \mathrm{muscle}$ area) and ratio of CD31/myocyte are shown in E and F. Data are mean \pm SEM from 10 fields per section (3 sections/mouse; $n=4$ for each strain). ${ }^{*} P<0.05$.

measured, and ischemic and nonischemic limb perfusion were measured before surgery and after surgery as indicated ( 3 days, 2 weeks, and 4 weeks after surgery; Figure 2A). Before surgery, the ratio of left leg to right leg gastrocnemius blood flow was 1 . After surgery, blood flow dropped by $80 \%$ and returned to a ratio of 1 in WT mice over 4 weeks. KO mice showed augmented recovery of hind limb perfusion and blood flow compared with WT mice at 2-4 weeks (Figure 2A). As demonstrated previously $(10,25)$, enhanced clinical recovery and limb perfusion could be due to increased arteriogenesis from existing vessels of the upper limb and increased neovascularization/vessel maturation in the lower limb. Consistently, ischemia-induced arteriogenesis in $\mathrm{KO}$ mice was significantly increased as measured by microfil casting followed by $\mathrm{x}$-ray angiogram analysis (Figure $2 \mathrm{~B}$ with quantification in Figure 2C) and 3D micro-CT $(10,25)$ (data not shown). Similar$l y$, angiogenesis and vessel maturation in $\mathrm{KO}$ mice were increased in gastrocnemius muscles as measured by immunostaining with anti-CD31 and anti- $\alpha$-SMA. After 2 weeks of ischemia, CD31positive capillaries surrounding the skeletal muscle fibers in WT mice were significantly increased (Figure 2D with quantification of capillary number $/ \mathrm{mm}^{2}$ and ratio of capillary/fiber in Figure 2 , E and F). Consistent with increased functionally of perfused vessel in $\mathrm{KO}$ mice, ischemic-induced vessel maturation was also increased in $\mathrm{KO}$ mice (Supplemental Figure 3).
The role of AIP1 in angiogenesis was further determined in a sponge granuloma model $(26,27)$. In the early phase (1-3 weeks), the invasion of the sponge by cells and the formation of new tissue permit the quantification of neovascularization. Polyvinyl alcohol sponges were implanted subcutaneously into WT and KO mice and harvested on days 14 or 21 . The sponges were subjected to trichrome staining. $\mathrm{KO}$ mice had increased invasion by cells and sponge encapsulation (Figure 3A, indicated by arrows). Moreover, the sponges from $\mathrm{KO}$ mice had dramatic increases in number of red blood cells (Figure 3A with quantification in Figure 3B), an indicator of new blood vessel formation. We further determined macrophage infiltration and blood vessel formation by immunostaining. KO mice had enhanced macrophage infiltration (measured by anti-F4/80 staining) and neovascularization (measured by antiCD31 staining) (Figure 3C with quantification in Figure 3, D and E). Interestingly, red blood cells in sponges from WT mice were localized inside vessels (arrowheads in Figure 3A). In contrast, red blood cells in sponges from $\mathrm{KO}$ mice were outside the vasculature (Figure 3A), suggesting enhanced vascular leakage. This observation prompted us to examine activation of VEGF-VEGFR2-mediated vascular leakage (15). Consistent with mice that underwent enhanced macrophage infiltration, KO mice had increased VEGF production as determined by Western blot with anti-VEGF (Figure 3F). Similarly, total and phosphorylated VEGFR2 in KO mice 
A

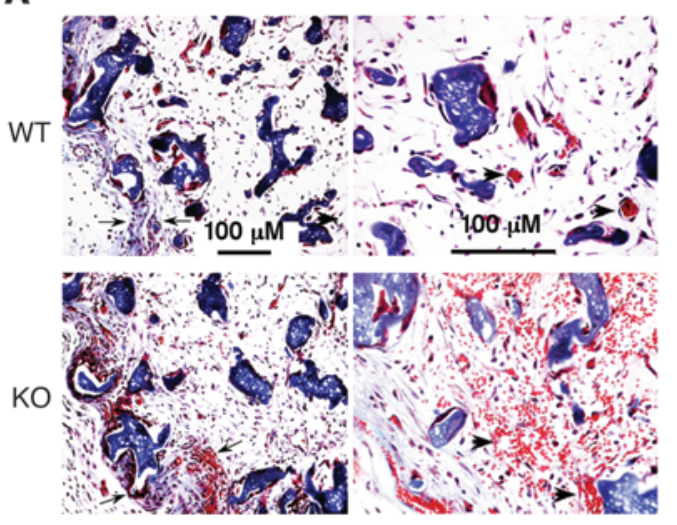

C
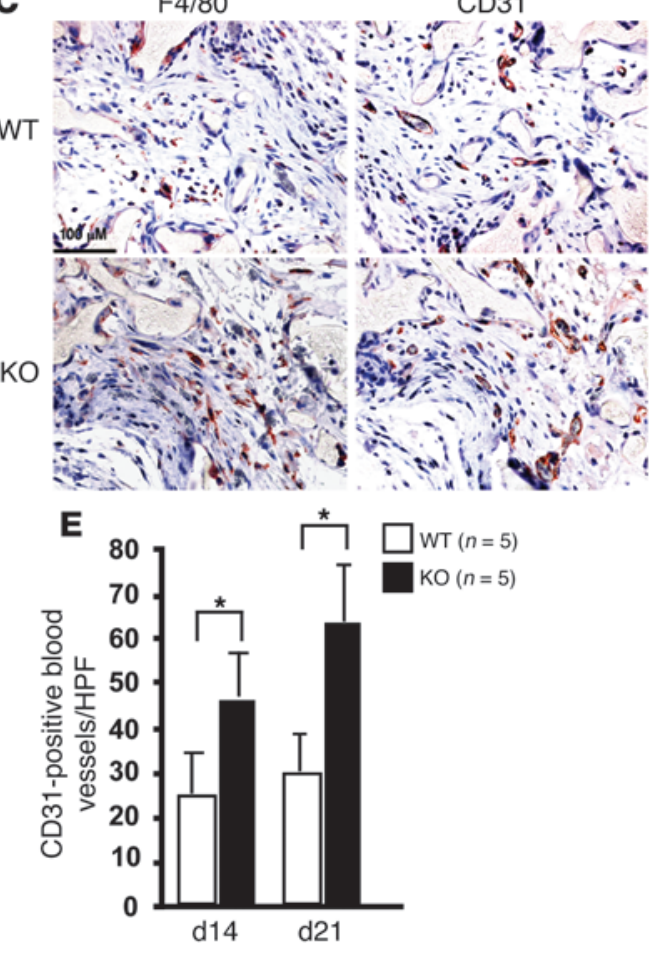

B

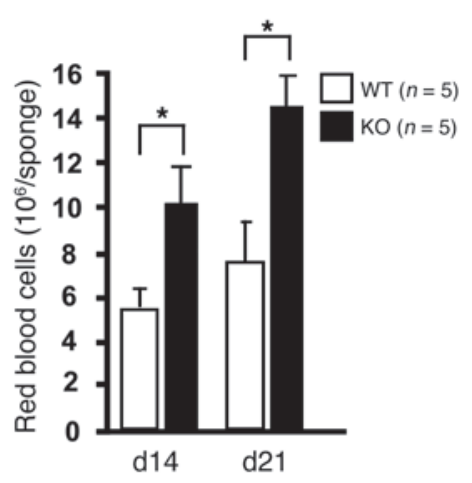

D

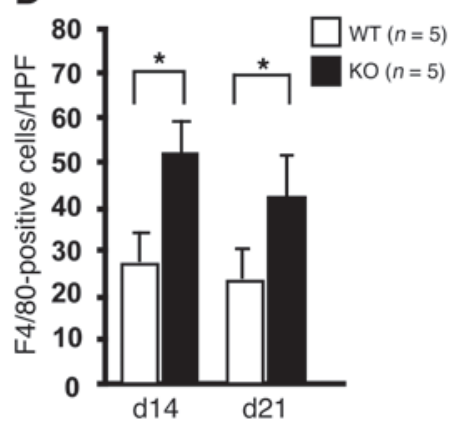

Figure 3

KO mice showed enhanced angiogenesis in a sponge granuloma model. A polyvinyl alcohol sponge was implanted subcutaneously into WT and KO mice. The sponges were harvested on day 21 and subjected to trichrome staining (A) and immunostaining for macrophage infiltration $(F 4 / 80)$ and blood vessel formation (anti-CD31) (C). (B) The number of red blood cells, (D) F4/80-positive cells, and (E) CD31-positive capillaries were quantified. Data are mean \pm SEM from 10 fields per section (3 sections/mouse and $n=5$ for each strain). ${ }^{*} P<0.05$. (F) Enhanced VEGF-VEGFR2 signaling in KO. Fluids and cells from sponges were harvested. VEGF from the fluid was determined by Western blot with anti-VEGF. Phosphorylated and total VEGFR2 proteins in cell lysates were determined by Western blot with anti-phosphoVEGFR2 (pY1054/59) and anti-VEGFR2, respectively. AIP1 and $\beta$-tubulin were also determined. HPF, high-power field. were also dramatically increased as detected by Western blot with a phospho-specific antibody (Figure $3 \mathrm{~F}$ ). These results suggest that KO mice had augmented VEGF-VEGFR2 signaling.

$V E G F-i n d u c e d$ ear, retina, and cornea neovascularization were greatly augmented in KO mice. The hind limb ischemia and the sponge granuloma models represent pathological settings, involving inflammation, multiple cytokines, and proangiogenic factors (28). To directly determine the role of AIP1 in regulating VEGF-VEGFR2dependent angiogenesis in vivo, we performed VEGF-induced retina, ear, and cornea angiogenesis in $\mathrm{KO}$ mice. For the retina angiogenesis, adenovirus encoding $\mathrm{VEGF}_{164}$ or $\beta$-gal was injected intravenously. Five days after viral injection, the vasculature was visualized by isolectin staining as described previously (29). VEGFinduced retina angiogenesis was significantly augmented in $\mathrm{KO}$ mice (Figure 4A with quantification in Figure 4B). For the ear angiogenesis model, adenovirus encoding $\mathrm{VEGF}_{164}$ or $\beta$-gal was injected intradermally into the ears. VEGF-induced angiogenesis was accessed by direct microscopy (Supplemental Figure 4A). Ear permeability was measured by Evans blue dye (EBD) assay (Supplemental Figure 4B). Ear vasculature was also visualized by a wholemount staining with PE-conjugated anti-CD31 (Supplemental Figure 4C with quantification in Supplemental Figure 4D). We also examined angiogenesis in the avascular cornea, into which recombinant VEGF protein formulated into Hydron pellets was implanted as described previously (30) (Figure 4, C and D). In these assays, VEGF-induced angiogenesis was dramatically augmented in KO mice. To determine if the loss of AIP1 is responsible for enhanced VEGF responses, AIP1 was reintroduced into retina of $\mathrm{KO}$ mice by an adenoviral expression system. AIP1 overexpression in KO mice diminished augmented VEGF responses (Supplemental Figure 5). These data suggest that AIP1 is critical for "normal" VEGF response and may function as a specific endogenous inhibitor in VEGF-induced angiogenesis.

$V E G F$-induced EC migration and tube formation were inhibited by AIP1 overexpression but augmented by AIP1 deficiency. Next we examined VEGF-dependent angiogenesis using in vitro models for EC 
A

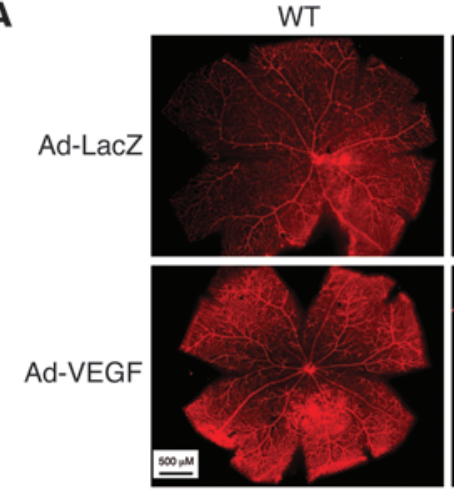

B

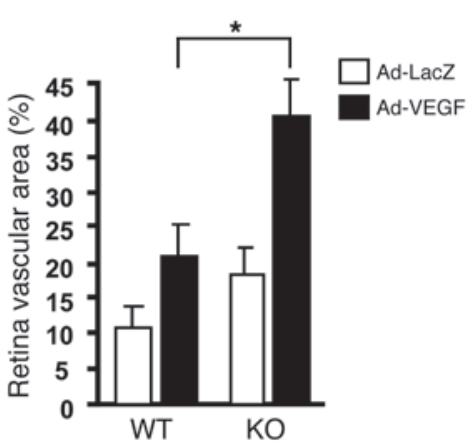

KO

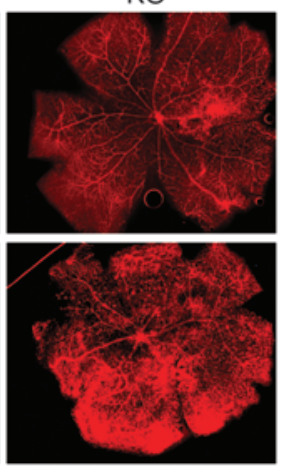

C $\quad W T$
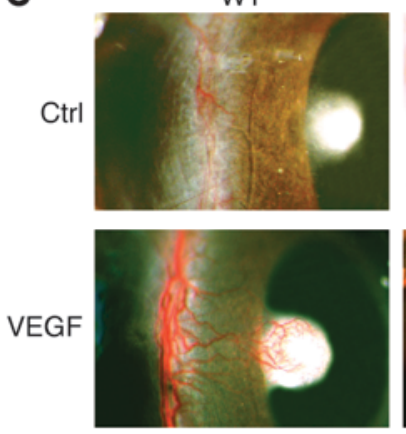

Ad-VEGF
WT
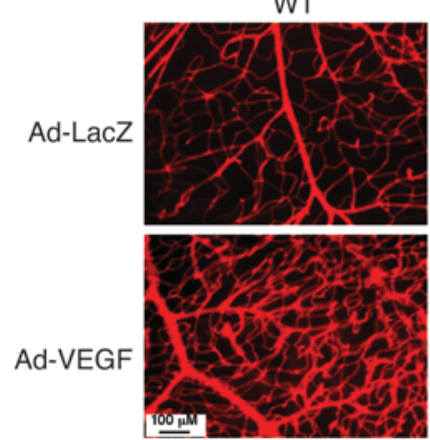

KO

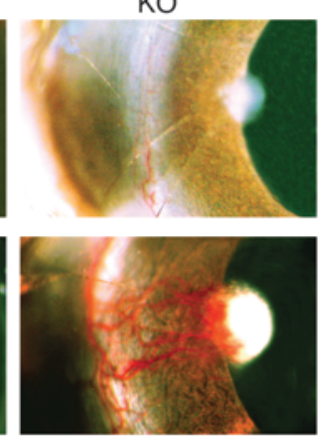

KO

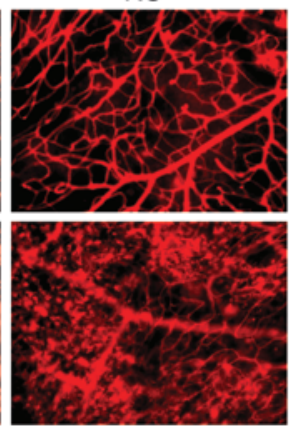

D

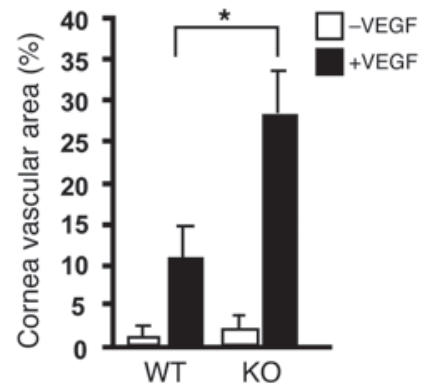

Figure 4

VEGF-induced retina and cornea neovascularization were greatly augmented in KO mice. (A and B) VEGF-induced retina angiogenesis. Ad-VEGF or Ad-LacZ was injected intravenously into WT and KO mice. Retina vasculature was visualized by isolectin staining shown in A, with quantification of vessel density in B. (C and D) VEGF-induced cornea angiogenesis assay. A Hydron pellet containing VEGF was implanted into the cornea of WT and KO mice. Angiogenesis was assessed by stereo microscopy on day 5 following implantation (C) and vascular density was quantified in $\mathbf{D}$. Data are mean \pm SEM from 10 fields per tissue (ear, retina, or cornea) $\left(n=5\right.$ for each group). ${ }^{*} P<0.05$. Ad-LacZ, adenoviral vector encoding the LacZ gene for expression of $\beta$-gal; Ad-VEGF, adenoviral vector encoding the VEGF gene for expression of VEGF.

migration and tube formation. Primary human ECs (HUVECs) were infected with an inducible adenoviral vector expressing AIP1 (Figure 5A), and the effects of AIP1 overexpression on EC migration were determined in a transwell migration assay (Figure 5B) and a monolayer "wound injury" assay (Figure 5, C and D). In both assays, VEGF-induced EC migration was markedly inhibited by AIP1 overexpression. The effects of AIP1 overexpression on EC tube formation were also determined in a matrigel tube assay. Similar to results from EC migration, AIP1 overexpression significantly inhibits EC tube formation (Figure 5, E and F). Conversely, the effects of AIP1 deficiency on EC angiogenesis were also determined in both AIP1 knockdown HUVECs by siRNA and mouse lung microvessel ECs isolated from KO mice. AIP1 deficiency strongly augmented EC migration and tube formation in identical assays (Figure 5, G-J, for mouse lung microvessel ECs; Supplemental Figure 6A for HUVEC). Similar results were obtained from mouse brain microvessel ECs (MBECs) isolated from KO mice.

AIP1 inhibits VEGFR2-dependent signaling by associating with an active form of VEGFR2 at the late phase of VEGF response. The results above prompted us to define the mechanisms by which AIP1 negatively regulates VEGFR2 signaling. To this end, WT and KO mouse ECs were treated with VEGF $(10 \mathrm{ng} / \mathrm{ml})$ for the indicated times (0-30 minutes; Figure 6A), and the effects of AIP1 deficiency on VEGFR2 signaling (phosphorylation of VEGFR2, PLC- $\gamma$, and Akt) were determined by Western blot with phospho-specific antibod- ies. As reported previously, phosphorylation of VEGFR2, Akt, and PLC- $\gamma$ peaked at 5 minutes after VEGF treatment (9). Consistent with the in vitro angiogenesis assays, VEGFR2-mediated activation of PLC- $\gamma$ and Akt was substantially enhanced and prolonged in $\mathrm{KO}$ mouse ECs (Figure 6A). Similar results were obtained in HUVECs by AIP1 siRNA knockdown (Supplemental Figure 6B). However, AIP1 deletion appeared to have no effect on bFGF-induced signaling (phosphorylation of PLC- $\gamma$ and Akt) (Supplemental Figure 7), suggesting that AIP1 may have a specific inhibitory effect on VEGF-induced angiogenic response. In contrast, AIP1 deficiency in KO cells showed a reduced response in TNF-induced JNK activation as we recently shown in the same cell line (31).

We then determined whether AIP1 inhibits VEGFR2 signaling by directly associating with VEGFR2 receptor complexes. To this end, HUVECs were treated with VEGF for various times (Figure $6 \mathrm{~B})$, and endogenous the AIP1-VEGFR2 complex was determined by a coimmunoprecipitation assay with anti-VEGFR2, followed by Western blot with anti-AIP1. Phosphorylation of VEGFR2 and formation of VEGFR2-PLC- $\gamma$ and VEGFR2-PI3K complexes peaked at 5 minutes in response to VEGF. However, the AIP1VEGFR2 complex was only weakly detected at 5 minutes, but dramatically increased after 15-30 minutes (Figure 6B). These data suggest that AIP1 is recruited to VEGFR2 at a late phase of VEGF response when activation of VEGFR2-PI3K/PLC- $\gamma$ declines. We reasoned that AIP1 associates with an active phosphorylated 
A

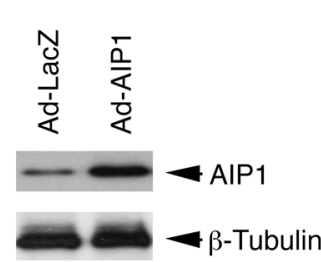

B

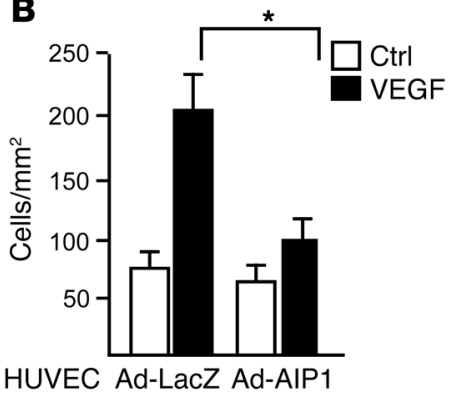

C

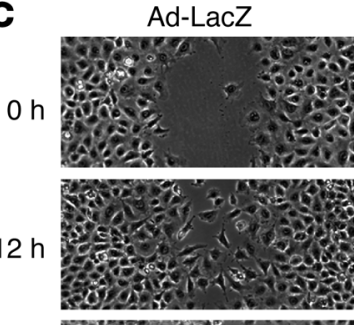

Ad-AIIP1

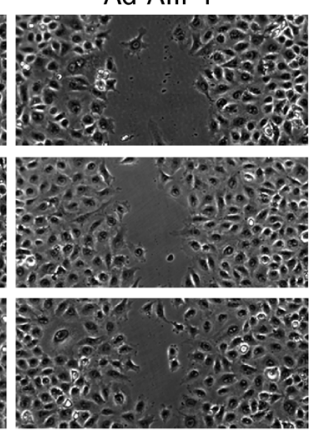

D

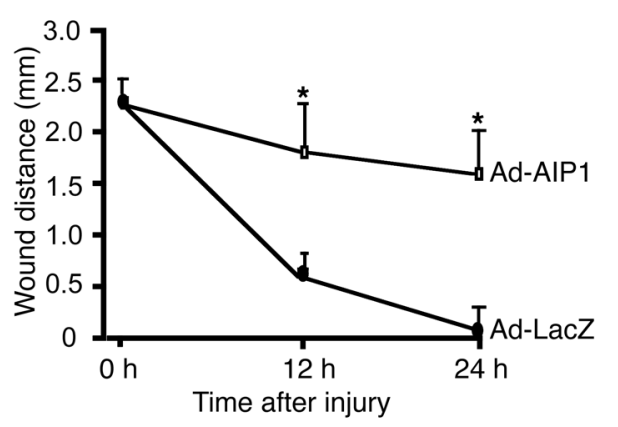

E

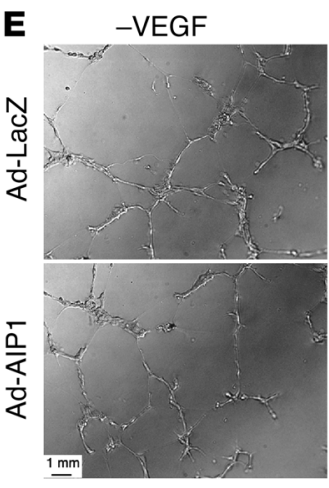

I

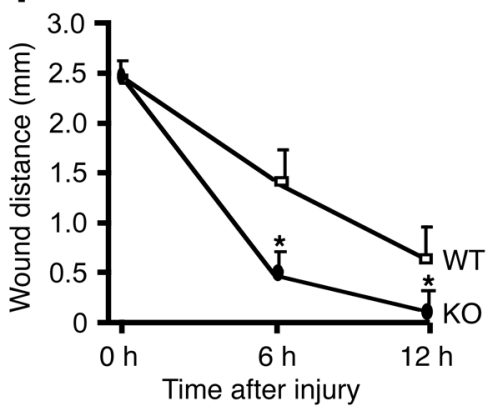

F

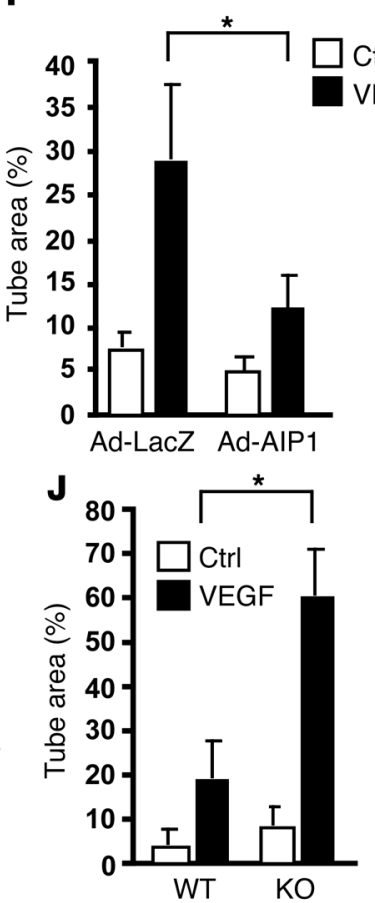

Figure 5

VEGF-induced EC migration and tube formation were inhibited by AIP1 overexpression but augmented by AIP1 deletion. (A-D) AIP1 overexpression inhibits EC migration. HUVECs were infected with adenovirus expressing WT (Ad-AIP1) in the presence of $4 \mu \mathrm{m}$ doxycycline for 24 hours. (A) AIP1 expression was determined by Western blot with anti-AIP1. (B) Cells were cultured in serum-free media for overnight and subjected to a transwell migration assay and (C with quantification in $\mathbf{D})$ monolayer "wound injury" assay in response to VEGF (10 ng/ml). (E and F) AIP1 overexpression inhibits EC tube formation. HUVECs were infected with Ad-AIP1 and subjected to a Matrigel tube formation assay VEGF $(10 \mathrm{ng} / \mathrm{ml})$ (E with quantification in $\mathbf{F})$. (G-J) AIP1 deficiency augmented on EC migration and tube formation. WT and KO mouse lung microvessel ECs (MLECs) were cultured in $0.5 \%$ FBS overnight and subjected to EC migration and tube formation as described above. (G) AIP1 expression was determined by Western blot with anti-AIP1. Quantification for EC migration in transwell assay (H), "wound injury" assay (I), and tube formation assay $(\mathbf{J})$ are shown. Data presented are mean \pm SEM of the triplicates from 3 independent experiments. ${ }^{*} P<0.05$.

form of VEGFR2. To test our hypothesis, ECs were treated with VEGF in the absence or presence of VEGFR2-specific inhibitor SU1498. Indeed, SU1498 specifically blocked phosphorylation of VEGFR2 as well as the formation of the AIP1-VEGFR2 complex (Figure 6C). We also examined the effects of PI3K-specific inhibitor LY294002, PLC- $\gamma$ inhibitor U73122, and its inactive homolog U73343 on VEGFR2-AIP1 association. As expected, LY294002 and U73122 specifically blocked VEGF-induced phospho-Akt and phospho-PLC- $\gamma$, respectively (Figure 6C). Interestingly, PI3K inhibitor (PLC- $\gamma$ inhibitor) strongly enhanced VEGF-induced association of AIP1 with VEGFR2 as well as the VEGFR2-PI3K p85 complex (Figure 6C). Similar results were obtained with another
PI3K-specific inhibitor, wortmannin (Figure 6D). It is conceivable that PI3K inhibition enhances the formation of VEGFR2-PI3KAIP1 complex. These data support that AIP1 is recruited to the VEGFR2-PI3K complex at a late phase of VEGF response, leading to an inhibition of VEGFR2-dependent signaling.

AIP1 via its proline-rich (PR) motif binds to the Src homology region 3 (SH3) domain of $\mathrm{PI} 3 \mathrm{~K}$, while through the $\mathrm{C} 2$ domain, AIP1 binds to a tyrosine-phosphorylated form of VEGFR2. We further investigated the VEGFR2-PI3K-AIP1 complex by mapping the critical domains of AIP1 for the interactions. AIP1 contains the N-terminal (AIP1-N) and C-terminal (AIP1-C) half and a PR motif at the C-terminal (AIP1-C-PR) half, which we predicted to bind to 
A

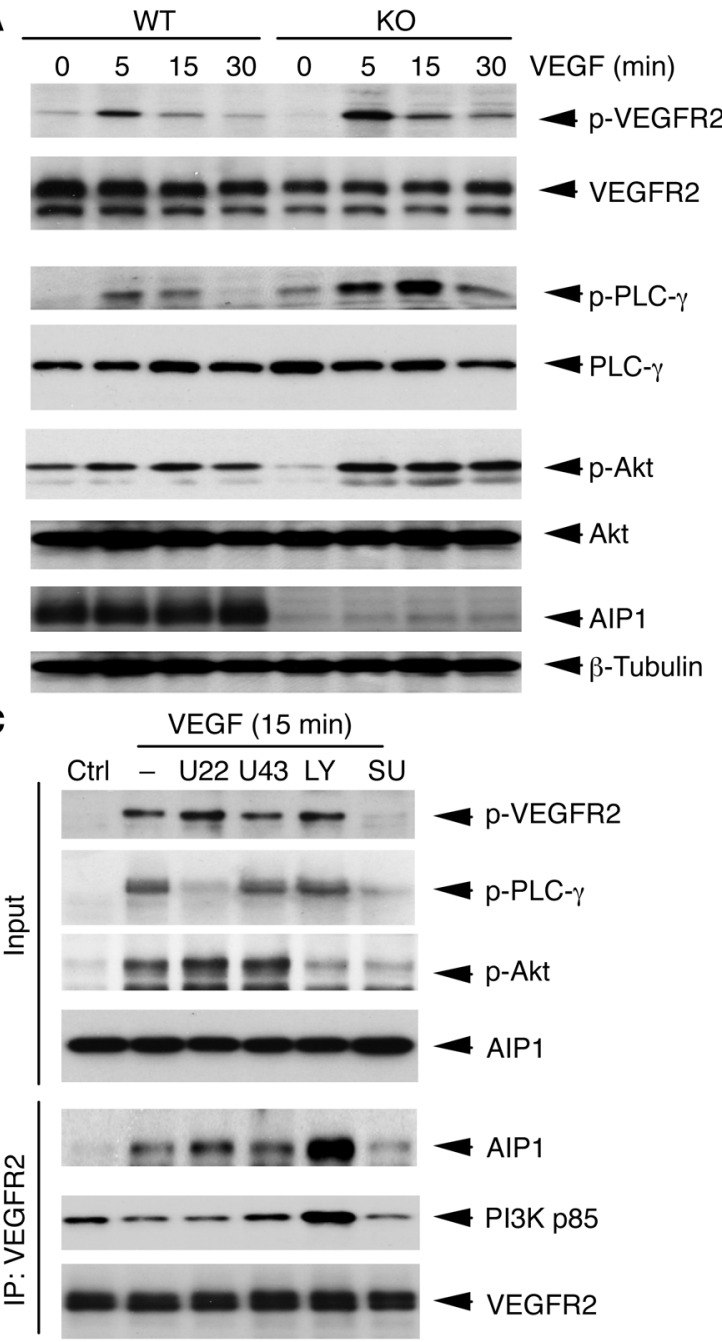

B
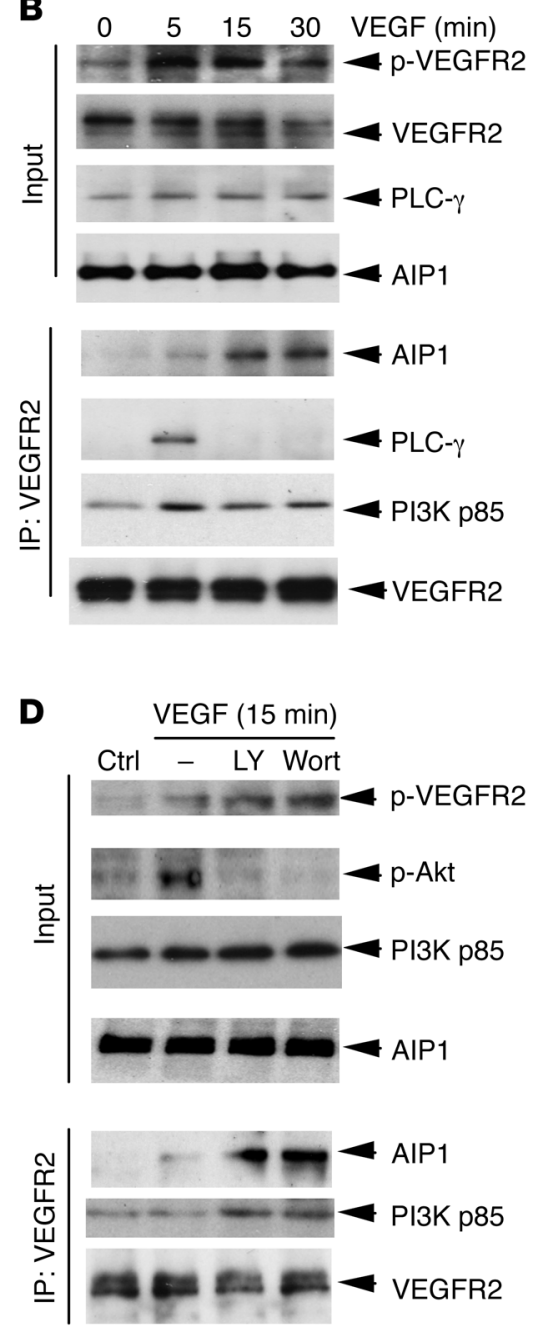

Figure 6

AIP1 inhibits VEGFR2-dependent signaling by associating with an active form of VEGFR2 in the late phase of VEGF response. (A) WT and KO mouse lung microvessel ECs were cultured overnight in 0.5\% FBS, followed by VEGF treatment (10 ng/ml) for 0-30 minutes as indicated. Phosphorylation of VEGFR2, PLC- $\gamma$, and Akt and the total proteins were determined by Western blot with respective antibodies. (B) Formation of the VEGFR2-PI3K-AIP1 complex. HUVECs were treated with VEGF for 0-30 minutes as indicated. Proteins of $p-V E G F R 2$, total VEGFR2, PLC- $\gamma$, and AIP1 in the input were determined by Western blot with respective antibodies. Association of endogenous VEGFR2 with AIP1, PLC- $\gamma$, and PI3K p85 was determined by immunoprecipitation with anti-VEGFR2, followed by Western blot with anti-AIP1, anti-PLC- $\gamma$, and PI3K p85, respectively. (C and D) Effects of inhibitors on the VEGFR2-PI3K-AIP1 complex formation. HUVECs were serum starved for 16 hours and pretreated with the following inhibitors: U73122 (U22), U73343 (U43), SU1498 (SU), or LY294002 (LY) (each at $10 \mu$ M for 30 minutes in C; LY294002 and wortmannin (wort) at $1 \mu \mathrm{M}$ for 30 minutes in D. -, mock. Cells were stimulated with VEGF $(10 \mathrm{ng} / \mathrm{ml})$ for 15 minutes, and proteins of p-VEGFR2, total VEGFR2, PLC- $\gamma$, and AIP1 in the input were determined by Western blot with respective antibodies. Association of endogenous VEGFR2 with AIP1 and PI3K p85 was determined as above.

the $\mathrm{SH} 3$ domain of the regulatory p85 subunit of PI3K (Figure 7A). We tested this possibility using an in vitro pulldown assay with glutathione-S-transferase-p85-SH3 (GST-p85-SH3) fusion protein as bait. GST-fusion proteins containing the SH3 domains from $\mathrm{Src}$ and $\alpha$-spectrin protein were used as controls. AIP1-C-PR containing the PR motif (Figure 7A) from 293T expression lysate strongly bound to PI3K p 85 and weakly bound to Src but not to spectrin. In contrast, AIP1-C with a deletion of the PR region (AIP1-C- $\triangle \mathrm{PR}$ ) was not pulled down by GST-SH3 of p85 (Figure 7B), confirming the PR motif on AIP1 for PI3K binding. To determine the critical domains of AIP1 for VEGFR2 binding, an expression vector encod- ing for a full-length AIP1 (AIP1-F), AIP1-N, or AIP1-C (Figure 7A) was coexpressed with VEGFR2, and AIP1-VEGFR2 association was determined by a coimmunoprecipitation assay. AIP1-N, but not AIP1-F or AIP1-C, bound to VEGFR2 (Figure 7C). AIP1-F did not associate with VEGFR2, consistent with our previous observations that AIP1 normally exists in a closed inactive form by an intramolecular interaction between the $\mathrm{N}$ - and the C-terminal domains (18). AIP1-N contains several structural domains: PH for the targeting on membrane, $\mathrm{C} 2$ for the interactions with proteins (e.g., ASK1), and GAP for the inhibition on Ras (Figure 7A). We then determined whether these domains of AIP1 are critical for associa- 
A

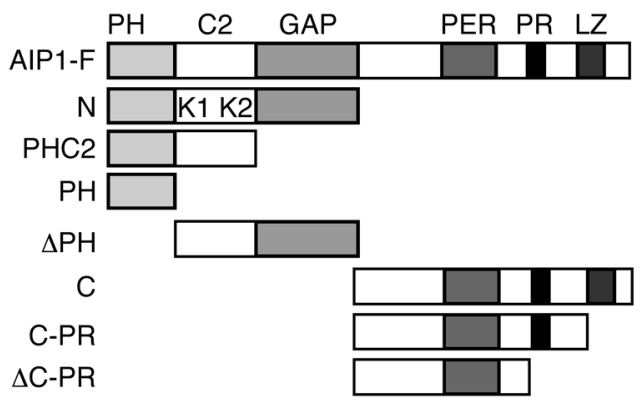

D

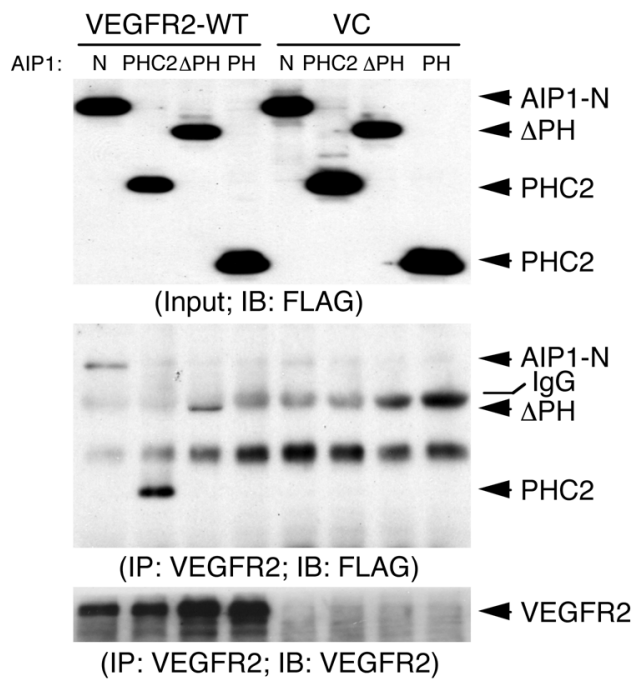

B

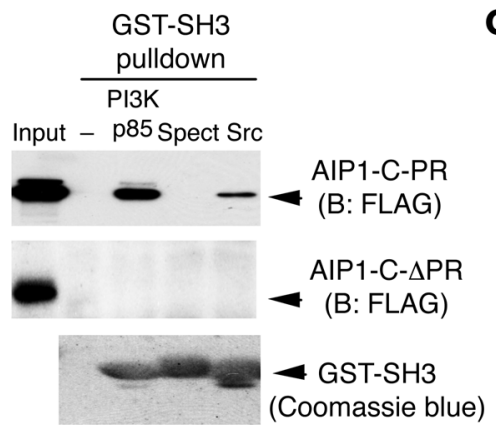

E

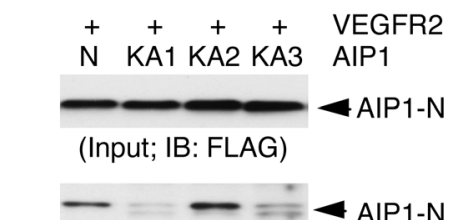

(IP: VEGFR2; IB: FLAG)

$0=0$ - 0 VEGFR2

(IP: VEGFR2; IB: VEGFR2)
C VEGFR2-WT $\frac{V C}{F N \text { N }}$

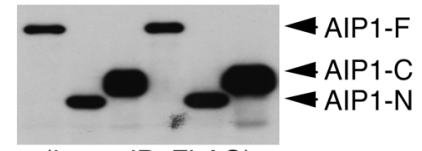

(Input; IB: FLAG)

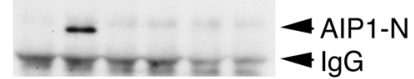

(IP: VEGFR2; IB: FLAG)

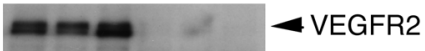

(IP: VEGFR2; IB: VEGFR2)

Figure 7

AIP1 via its PR motif binds to the SH3 domain of PI3K, while through the C2 domain AIP1 binds to a tyrosine-phosphorylated form of VEGFR2. (A) Schematic diagram for AIP1 structural domains and expression constructs. PH indicates PH domain. C2, PKC conserved domain where K1 and K2 indicate the 2 lysine clusters; PER, period-like domain; LZ, leucine-zipper motif; $\triangle \mathrm{PR}$, deletion of the PR; N, AIP1-N; C, AIP1-C; C-PR, AIP1 containing PR motif at the C-terminal. (B) In vitro GST pulldown assay. AIP1-C-PR and AIP1-C- $\Delta$ PR from 293T expression lysate were used for a pulldown assay with GST-fusion proteins containing the SH3 domains from PI3K p85, $\alpha$-spectrin (Spect), and Src kinase. Bound AIP1-C-PR and AIP1-C- $\triangle$ PR were determined by Western blot with anti-FLAG. GST-SH3 fusion proteins were visualized by a Coomassie blue staining. ( $C$ and $\mathbf{D}$ ) Various AIP1 constructs (F, AIP1-F; N, AIP1-N; C, AIP1-C) were coexpressed with VEGFR2, and AIP1-VEGFR2 association was determined by immunoprecipitation with anti-VEGFR2, followed by Western blot with anti-FLAG. (E) A critical role the lysine cluster within the AIP1 C2 domain. AIP1-N or its mutant in the C2 domain (KA1-3) was transfected into 293T in the presence of VEGFR2. AIP1-VEGFR2 association was determined as above.

tion with VEGFR2. Results showed that AIP1-N, PHC2, and $\triangle \mathrm{PH}$, but not AIP1-PH, associated with VEGFR2 in a coimmunoprecipitation assay. These data suggest that the $\mathrm{C} 2$ domain of AIP1 is critical for VEGFR2 binding (Figure 7D).

The positive charge residues with the $\mathrm{C} 2$ domain are critical for protein-protein interactions. We analyzed the effects of mutations of the 2 lysine clusters (Figure 7A), between which the second lysine cluster has been shown to be important for ASK1 binding (18). Results showed that a mutation at the first lysine cluster (KA1), but not that at the second cluster (KA2), reduced the binding of AIP1 to VEGFR2. KA3 contains both mutations and did not show further reduction in the binding (Figure 7E). These data strongly support that the AIP1 via its first lysine cluster associates with VEGFR2.

Effects of AIP1 mutants on p-VEGFR2-PI3K-Akt signaling. Finally, we determined a direct inhibitory effect of various AIP1 mutants on VEGFR2-dependent signaling. To this end, VEGFR2 was coexpressed with an AIP1 mutant (AIP1-N, AIP1-PR, PHC2) in 293T cells that do not express endogenous VEGFR2. Overexpression of VEGFR2 (but not the kinase-inactive form) induced autophosphorylation as well as activation of downstream targets PI3KAkt and PLC- $\gamma$ as determined by Western blot with phospho-specific antibodies (Figure 8A). Coexpression of AIP1-N and PHC2 containing the $\mathrm{C} 2$ domain strongly inhibits phopsho-VEGFR2 and its downstream targets phospho-Akt and phospho-PLC- $\gamma$. AIP1-PHC2 contains a deletion of the GAP1 domain, suggesting that the GAP activity is not required for the inhibition of AIP1 on VEGFR2. We further showed that AIP1-N-R289L, with a mutation at the catalytic site, similar to AIP1-N, could bind to VEGFR2 and inhibit phosphorylation of VEGFR2 (Figure 8B). In contrast, AIP1-C-PR specifically blocked VEGFR2-inudced phospho-Akt but not phospho-VEGFR2 or phospho-PLC- $\gamma$ 
A

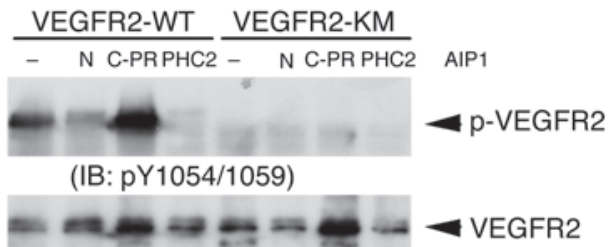

(IB: VEGFR2)

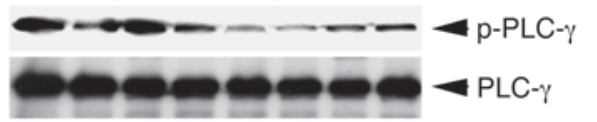

$\begin{array}{llllllll}1.0 & 0.3 & 1.0 & 0.3 & 0.1 & 0.1 & 0.1 & 0.15 \\ \text { (p-PLC- } \gamma / \text { PLC- } \gamma)\end{array}$

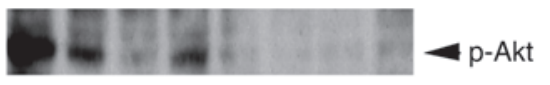

(IB: p-Akt)

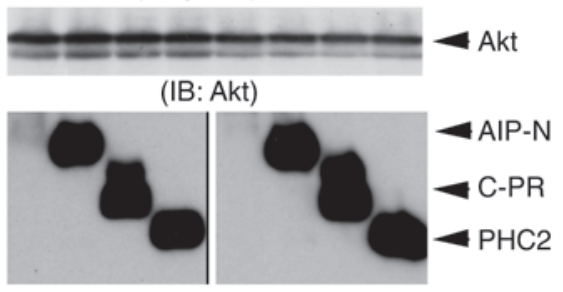

(IB: FLAG)

C

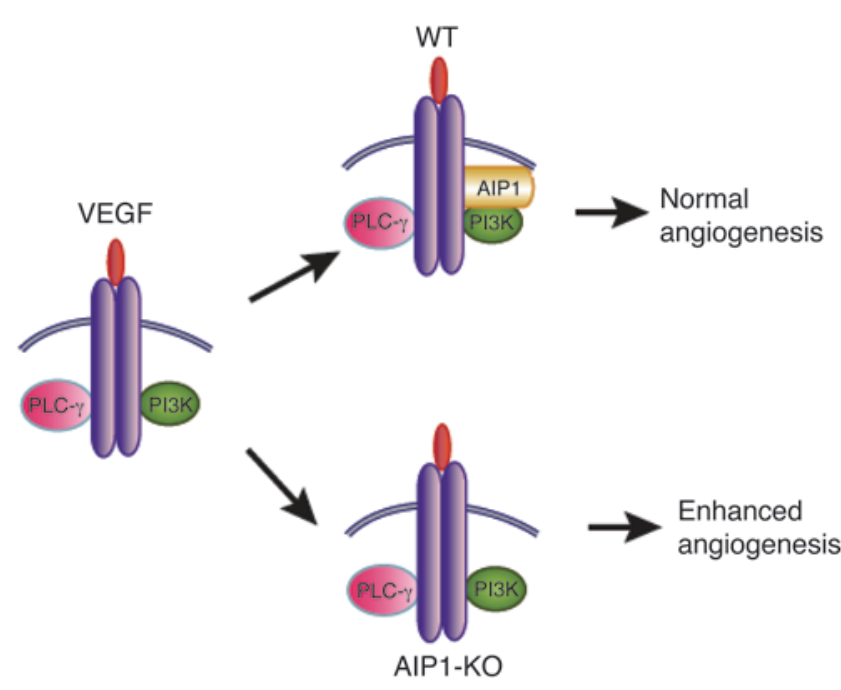

\section{Figure 8}

Mechanism for a negative regulation of VEGFR2 by AIP1. (A and B) Effects of AIP1 mutants on VEGFR2-mediated signaling. VEGFR2-WT or a kinaseinactive mutant $(\mathrm{KM})$ was cotransfected with various AIP1 mutants (AIP1-N, AIP1-C-PR, and PHC2 in A and AIP1-N and AIP1-N-R289L [RL] in B) into 293T cells. Phospho-VEGFR2 (pY1054/1059) and total VEGFR2, PLC- $\gamma$, and Akt were determined by respective antibodies. Relative ratios of $p$-VEGFR2/VEGFR2, p-PLC- $\gamma /$ PLC- $\gamma$, and p-Akt/Akt are shown, with untreated WT as 1.0. Expression of AIP1 mutants was determined by Western blot with anti-FLAG. Note that the 2 FLAG blots were run on the same gel but noncontiguously as indicated by a black line. Association of AIP1 with various VEGFR2 was determined by immunoprecipitation with anti-VEGFR2, followed by Western blot with anti-FLAG. (C) A model for AIP1 as an endogenous regulator in VEGFR2-mediated signaling. VEGF rapidly induces VEGFR2 dimerization and autophosphorylation, followed by the recruitment and activation of PLC- $\gamma$ and PI3K, leading to activation of PLC- $\gamma-E R K, P I 3 K / A k t$, and angiogenesis. AIP1 is recruited to the VEGFR2-PI3K complex in response to VEGF response. AIP1 via its C2 domain associates with VEGFR2 while via its $P R$ domain binds to the $\mathrm{SH} 3$ domain of PI3K p85 to switch off VEGFR2 activation, leading to normal angiogenesis. In the absence of AIP1, VEGFR2-mediated angiogenic responses are enhanced.
(Figure 8A). These results strongly support that AIP1 via different domains targets both VEGFR2 and PI3K p85, leading to inhibition of VEGFR2-dependent angiogenic signaling.

\section{Discussion}

In the present study, we show that KO mice exhibit dramatically enhanced ischemia and inflammatory angiogenesis in several in vivo models (ischemic hind limb and sponge granuloma models). Furthermore, VEGF-induced neovascularization is greatly augmented in $\mathrm{KO}$ mice, which can be suppressed by re-expression of AIP1. In cultured ECs, overexpression of AIP1 inhibits, while knockout or knockdown of AIP1 in ECs enhances, VEGF-induced migration and tube formation. Mechanistic studies suggest that AIP1 is recruited to the VEGFR2-PI3K complex at a late phase of VEGF response. AIP1 via its C2 domain associates with VEGFR2 while via its PR domain binds to the $\mathrm{SH} 3$ domain of PI3K p85, leading to an inhibition of VEGFR2-mediated angiogenic signal- ing (Figure 8C). Our in vivo and in vitro studies demonstrate that AIP1 functions as an endogenous scaffold in inflammatory angiogenesis by suppressing VEGFR2-Akt-dependent signaling.

The ischemic hind limb model was created to study peripheral arterial disease and has been extensively used to assess a role of a gene in adult arteriogenesis and angiogenesis. The model represents pathological settings, involving inflammation, cytokine/ proangiogenic factors, and altered systemic/local blood flow (28). We first assessed the phenotype of $\mathrm{KO}$ mice in this model, in which $\mathrm{KO}$ mice showed impaired arteriogenesis and angiogenesis. We then determined the role of AIP1 in more "simplified" in vivo angiogenesis models, in which only limited components were involved. For example, inflammation (but not ischemia) is predominant in the sponge granuloma model (26). In contrast, less inflammation is involved in the ear and retina angiogenesis assay, in which a single proangiogenic factor could be applied. In these assays, we have shown that AIP1 specifically inhibits 
adenoviral-expressed VEGF-induced angiogenesis in the ear and retina angiogenesis assays. Moreover, recombinant VEGF-induced cornea angiogenesis is also augmented in $\mathrm{KO}$ mice.

Our study here demonstrates that AIP1 is dispensable in vascular development but suppresses VEGF-induced angiogenesis in adult tissues using these animal models. The mechanisms by which $\mathrm{KO}$ mice show normal development, birth, growth, and breeding compared with WT mice are not known. The close members of RasGAP family include $\mathrm{p} 120 \mathrm{Ras}$ GAP and nGAP (Ras protein activatorlike 1). It has been reported that deletion of p120RasGAP in mice results in early embryonic lethality associated with defective vasculogenesis and increased apoptosis of neuronal cells, indicating the importance of this gene during vascular embryogenesis (32). Whether or not these Ras-GAP proteins compensate AIP1 function in $\mathrm{KO}$ mice needs further investigation. Previously, several genetically knockout mice that are developmentally "normal" have been used to test the genes in regulating postnatal angiogenesis and remodeling. The tested genes include the VEGF homolog placental growth factor $(33)$ and Akt $(34,35)$ among others. Embryonic angiogenesis in mice was not affected by the deficiency of these genes. However, loss of these genes impaired ischemic-induced angiogenesis. Our recent studies indicate that TNF receptor 2 (TNFR2) and its downstream nonreceptor tyrosine kinase $B m x$ are additional members in this group of genes $(10,25)$. In contrast, deletion of antiangiogenic genes augmented angiogenesis in these models. For example, deletion of thrombospondin-2, the secreted extracellular matrix glycoprotein that functions as modulator of cell-matrix interactions, exhibits enhanced angiogenesis in a sponge granuloma model (27). Interestingly, KO mice show similar augmented angiogenic phenotype as TNFR1-KO mice as we described recently (25). This is consistent with our in vitro studies that AIP1 associates with TNFR1 and mediates TNFR1-induced activation of ASK1-JNK apoptotic signaling in ECs. Moreover, both KO and TNFR1-KO mice show enhanced VEGFR2 signaling. Given that TNF and VEGF are coexpressed in several inflammatory angiogenic settings. It is conceivable that both reduced ASK1-JNK apoptotic signaling and increased VEGFR2-PI3K-Aktmediated survival and migratory pathways lead to augmented angiogenesis in $\mathrm{KO}$ mice. However, our recent data suggest that AIP1 specifically mediates the TNF and ER stress-induced, but not oxidative stress-induced, ASK1-JNK pathway in ECs (31). Consistent with this finding, we did not detect dramatic differences between WT and KO mice in ischemia-induced ASK1-JNK activities (Supplemental Figure 8). It is likely that AIP1 primarily targets the VEGFR2-PI3K pathway to regulate ischemia-mediated arteriogenesis and angiogenesis.

Therefore, the most important finding of our present study is that we identified AIP1 as an endogenous inhibitor of VEGFR2mediated angiogenic signaling. Several positive regulators of VEGFR2 signaling have been reported, including co-receptor neuropilins, adhesion molecule VE-cadherin, and integrins. More recently, IQGAP, a Ras-GAP domain-containing protein, has been shown to interact with VEGFR2 and positively regulate VEGFR2 signaling (36). IQGAP1 was identified as a widely expressed IQ domain-containing protein, with a region containing sequence similarity to the Ras GTPase-activating proteins. However, subsequent in vitro analysis revealed that IQGAP1 actually binds to active GTP-bound Rac1 and inhibits Rac intrinsic GTPase activity, therefore increasing Rac1 activity $(37,38)$. Thus, IQGAP, by activating Rac1, positively regulates VEGF-induced EC migra- tion. Consistently, IQGAP is colocalized with phosphorylated VEGFR2 at the leading edge of migrating ECs (36). In contrast, AIP1 associates with VEGFR2 and inhibits VEGFR2 activity and the downstream signaling. It needs to be determined if AIP1 antagonizes IQGAP1 function in VEGF signaling. Previous in vitro studies suggest that AIP1 is a Ras-GAP, which promotes Ras intrinsic GTPase activity, promoting a turn off of Ras signaling (17). However, PLC- $\gamma$ mediates activation of both PKC and ERK1/2 pathways in VEGFR2 signaling, contributing to EC proliferation. Thus, it has been proposed that VEGFR2 does not use the Ras pathway as a major downstream signaling pathway in the activation of ERK1/2 and DNA synthesis $(2,39,40)$. Consistently, our data show that AIP1-PHC2 (deletion of the GAP1 domain) or AIP1-N-R289L (mutation at the catalytic site) can still bind to VEGFR2 and inhibit VEGFR2 activity (Figure 8B), suggesting that the Ras-GAP activity of AIP1 is not required for its inhibitory effect on VEGFR2 signaling.

The exact mechanism by which AIP1 negatively regulates VEGFR2 activity needs further investigation. Nevertheless, our data support that AIP1 may directly bind to and inhibit VEGFR2 activity. Association of AIP1 with VEGFR2 is diminished by a VEGFR2-specific inhibitor, indicating that VEGFR2 signaling is required for the recruitment of AIP1. In contrast, AIP1-VEGFR2 as well as AIP1-PI3K and VEGFR2-PI3K complexes are strongly enhanced by a PI3K inhibitor. These results may support a model in which AIP1 retains VEGFR2-PI3K in an inactive state.

Negative regulation of VEGFR2 has been previously reported. VEGFR2 protein can be downregulated by E3 ubiquitin ligase c-Cbl-dependent ubiquitination and by PKC-dependent phosphorylation $(41,42)$. Although VE-cadherin functions as a potential co-receptor of VEGFR2 (12), it has also been implicated in cell contact-dependent inhibition of EC growth through reduced sensitivity of VEGF signaling. Further studies suggest that VE-cadherin, by recruiting a high cell density-enhanced phosphatase (DEP1) to the VEGFR2-VE-cadherin-catenin complex at cell-cell contacts, reduces VEGFR2 phosphorylation and ERK1/2 signaling (43). Similarly, a human low molecular weight phosphotyrosine phosphatase (HCPTPA) has been shown to directly associate with VEGFR2, and overexpression of HCPTPA in HUVECs leads to suppression of VEGF-induced cellular responses, suggesting that VEGFR2 might be a direct substrate of these phosphatases (44). It needs to be determined if AIP1 may recruit c-Cbl or VEGFR2-specific phosphatase(s), turning off VEGFR2 signaling.

Collectively, our in vivo and in vitro studies demonstrate that AIP1 functions as an endogenous inhibitor in VEGFR2 signaling. Our study suggests that AIP1 may be a novel target for the treatment of vascular diseases such as coronary artery and peripheral arterial diseases.

\section{Methods}

\section{Targeted inactivation of AIP1 gene by homologous recombination}

We have recently reported the KO strategy (31). Briefly, the AIP1 targeting arms were isolated from a BAC clone identified by screening a BAC library from Research Genetics (Invitrogen), using AIP1 cDNA as a probe. The targeting vector was constructed in pEASYflox backbone to contain a loxP site inserted upstream of AIP1 exon 5 and a neomycin cassette (Neo), flanked by 2 loxP sites downstream of exon 6 , using standard molecular procedures. The linearized targeting construct was electroporated into 129/C57B/6 ES cells, and the targeted clones were 
selected with G418 and ganciclovir. Resistant clones were screened for homologous recombination by PCR and confirmed by Southern blot analysis. Two independent $A I P 1^{+/ l o x}$ ES clones were injected into WT blastocysts. Chimeras were further bred with WT females for germline transmission. AIP $1^{+/ l o x}$ mice were mated with $\beta$-actin Cre mice to generate $A I P 1^{+/-}$mice with a deletion of both the targeting region (the exons $5 / 6)$ and the Neo gene. KO mice were obtained by mating between the heterozygous $\left(A I P 1^{+/-}\right)$male and female.

\section{In vivo angiogenesis models}

All animal studies were approved by the institutional animal care and use committees of Yale University. Eight- to twelve-week-old male littermates of WT and KO mice were used for all experiments.

Mouse hind limb ischemic model. The mouse hind limb ischemia model was used as described recently $(10,25)$. Briefly, following anesthesia (79.5 mg/kg ketamine; $9.1 \mathrm{mg} / \mathrm{kg}$ xylazine), the left femoral artery was exposed under a dissection microscope. The proximal of femoral artery and the distal portion of saphenous artery were ligated. All branches between these 2 sites were ligated or cauterized, and arteriectomy was performed. The sham operation was without any femoral artery ligation but skin incision. Blood flow was measured by PeriFlux system with Laser Doppler Perfusion Module (LDPU) unit (Perimed Inc.). A deep measurement probe was placed directly on gastrocnemius muscle to ensure a deep muscle blood flow measurement. Ischemic and nonischemic limb perfusion was measured before and 3 days, 2 weeks, and 4 weeks after surgery. The final blood flow values were expressed as the ratio of ischemic to nonischemic hind limb perfusion. Arteriogenesis was also analyzed by quantitative angiography (45). Briefly, 2 and 4 weeks after surgery, mice were anesthetized and heparinized. Mice were perfused with PBS containing vasodilators $(4 \mathrm{mg} / \mathrm{l}$ papaverine; $1 \mathrm{~g} / \mathrm{l}$ adenosine) for 3 minutes at physiological pressure through the descending aorta, and blood was drained from the inferior vena cava. The vasculature was fixed with $2 \%$ paraformaldehyde (in PBS) for 5 minutes, flushed with PBS for 2 minutes, and infused with contrast agent (bismuth oxychloride in saline and 10\% gelatin in PBS; $1: 1$ ). Mice were then immersed in ice to solidify the contrast agent. Microangiography was taken with Faxitron $\mathrm{x}$-ray machine (Hewlett-Packard) at $25 \mathrm{kV}$ and $3.25 \mathrm{~mA}$ for 3 minutes. Upper limb vascular density (pixel density), vessel length (average length of vessels with diameter $>1$ pixel), and fractal dimension were analyzed by modified ImageJ software (http://rsbweb.nih.gov/ij/) and MATLAB software (MathWorks).

Sponge granuloma model. A polyvinyl alcohol sponge (Ultracell PVA sponge; Ultracell Medical Technologies Inc.) was implanted subcutaneously into 8- to 10-week-old WT and KO mice. Implants were excised at 2 and 3 weeks following implantation for histological analysis and cell recovery. We chose 2-3 weeks because inflammatory angiogenesis peaks during this period whereas the foreign body response begins at 4 weeks (46). Mice were monitored during this time, but they were not be subjected to any additional procedures. The sponge was harvested, fixed in $1 \%$ paraformaldehyde for 4.5 hours on ice, and embedded in paraffin. The paraffin section $(5 \mu \mathrm{M})$ was used for immunostain with anti-CD31 and -CD34 for angiogenesis and anti-F4/80 and -CD3 for inflammation. Stained cells were counted in several high-power fields (>20/animals), and cells per $\mathrm{mm}^{2}$ were presented.

Mouse cornea angiogenesis assay. Recombinant human $\mathrm{VEGF}_{165}$ (provided by $\mathrm{NIH}$ ) was formulated into Hydron pellets at $1 \mu \mathrm{g} / \mathrm{ml}$ and implanted into the cornea of WT and KO mice as we described previously (30). Angiogenesis was assessed by stereo microscopy on day 5 following implantation, the day of maximum angiogenesis. The contiguous circumferential zone of neovascularization was measured as clock hours, using the eye as a
360 degree circle and each 30 degree section as 1 clock hour. Alternatively, vascular area was measured. Animals receiving no treatment were used as positive controls. Implanted Hydron pellets containing no angiogenic factor served as negative controls.

Mouse ear and retina angiogenesis assay. For ear model, adenovirus encoding $\operatorname{VEGF}_{164}\left(1 \times 10^{9} \mathrm{PFU}\right)$ or same amount of control virus (adenoviral vector encoding the $L a c Z$ gene for expression of $\beta$-gal [Ad-LacZ]) was intradermally injected into the mice right and left ear skin, respectively. For retina model, adenoviral vector encoding the $V E G F$ gene for expression of VEGF (Ad-VEGF) and Ad-LacZ $\left(1 \times 10^{8} \mathrm{PFU}\right)$ were injected intravenously to right and left eyes, respectively. Five days after viral injection, animals were perfused with PBS and $4 \%$ paraformaldehyde/PBS. The ears (or retinas) were removed and stained with PE-conjugated anti-CD31 to visualize the vascular network. Vascular area was quantified.

EBD permeability assay. Five days after viral administration, EBD $(100 \mu \mathrm{l}$ of a $1 \%$ solution in $0.9 \% \mathrm{NaCl}$; Sigma-Aldrich) was injected into the retroorbital plexus of anesthetized WT and KO mice. Thirty minutes after the injection, mice were sacrificed and perfused with PBS through the left ventricle to clear the dye from the vascular volume. Ears were removed, dried in $60^{\circ} \mathrm{C}$ overnight, and weighed before EBD extraction using $1 \mathrm{ml}$ formamide at $55^{\circ} \mathrm{C}$ for 16 hours. EBD content was quantified by reading at $630 \mathrm{~nm}$ in a spectrophotometer as described previously $(47,48)$.

\section{Histology and immunohistochemistry}

Mice were sacrificed and tissues were perfused in situ with PBS and then inflated and fixed with $10 \%$ buffered formalin. The sections were paraffin embedded, sectioned in $5 \mu \mathrm{M}$, and stained with routine H\&E. Tissue sections were also stained with certain antibodies (e.g., anti-CD31, anti- $\alpha$-SMA, and anti-F4/80) as described previously $(10,25)$. Bound primary antibodies were detected using avidin-biotin-peroxidase (NovaRED Substrate Kit; Vector Laboratories). Pictures from 4 random areas of each section and 5 sections per mouse were taken using a Kodak digital camera mounted on a light microscope $(x 40$ objective). Images were quantified using the MATLAB software (The MathWorks) as described recently $(10,25)$. TUNEL assay was performed according to the instruction provided by the manufacturer's protocol (Roche) (25).

\section{Plasmids and adenovirus}

Mammalian expression plasmids for VEGFR2 (Flk-1/KDR) and mutants were described previously (9). Adenoviruses expressing LacZ and VEGF were generated in our laboratory. To construct AIP1 in an inducible adenovirus system, 2 vectors, pShuttleTetTripLac-Bam and pAdEasyTet-ONrev, were obtained from G. Akusjärvi (Uppsala University, Uppsala, Sweden). First, 2 pairs of primers, 5'-GATCCGCGGCCGCTCGCGACGATCGTCTAGATATCA-3' and 5'-GATCTGATATCTAGACGATCGTCGCGAGCGGCCGCG-3', were annealed and ligated into BamHI digested pShuttleTetTripLac-Bam to generate pShuttleTetTripLac-Bam-MCS, which contains multiple cloning sites. Two pairs of primers, 5BCLFLAG, 5'-GGTGATCAGCCACCATGGACTACAAGGACG-3' and 3XBAAIP, 5'CGTCTAGATTAACAATTGCTGCTGTTTCTGAACTC-3', were used to amplify AIP1-F by PCR using AIP1/2AB as a template. The PCR fragments were digested by $B c l \mathrm{I}$ and $\mathrm{XbaI}$ and ligated to the same enzyme-digested pShuttleTetTripLac-Bam-MCS. The resulting construct containing FLAGtagged AIP1-F in the Shuttle vector were designated pShuttleTetTripLacAIP1. pShuttleTetTripLac-AIP1 and pAdEasy-Tet-ONrev were cotransfected into BJ5183 to generate recombinant pAd-AIP1. pAd-AIP1 plasmid was further amplified in recombinant-deficient XL10-Gold strain (Stratagene). pAd-AIP1 virus was amplified in $293 \mathrm{~T}$ cells and purified by ultracentrifugation. Expression of AIP1 was induced by adding $4 \mu \mathrm{m}$ doxycycline into pAd-AIP1 infected cells for 16 hours (49). 


\section{Cell culture, inbibitors, cytokines, and transfection}

HUVECs were from Yale Endothelial Cell Facility (Yale University). Bovine aortic ECs were purchased from Clonetics and were cultured in DMEM medium containing 10\% FBS. Mouse EC isolation from muscle and lung tissues was performed as we described previously (25). Isolation of primary mouse brain endothelial cells was performed as follows. Meninges of 3-week-old mouse was carefully removed from the forebrain and gray matter was minced and then digested with $1 \mathrm{mg} / \mathrm{ml}$ collagenase CLS2 (Worthington) in DMEM (Sigma-Aldrich) containing $50 \mathrm{mg} / \mathrm{ml}$ gentamicin and $2 \mathrm{mM}$ glutamine in a shaker for 2 hours at $37^{\circ} \mathrm{C}$. The cell pellet was separated by centrifugation in $20 \%$ BSA-DMEM $(1,000 \times \mathrm{g}$, 20 minutes). The microvessels obtained in the pellet were further digested with $1 \mathrm{mg} / \mathrm{ml}$ collagenase-dispase (Roche) in DMEM for 1.5 hours at $37^{\circ} \mathrm{C}$. Microvessel endothelial cell clusters were separated on a $33 \%$ continuous Percoll gradient and washed twice in DMEM before planting on collagen type IV and human fibronectin-coated 35- $\mathrm{mm}$ plastic dishes. Cultures were maintained in DMEM supplemented with $20 \%$ FBS and $1 \mathrm{ng} / \mathrm{ml}$ bFGF (Roche). When the cultures reached $80 \%$ confluency (5-6 days in vitro), the endothelial cells were passed by brief treatment with trypsin $(0.05 \% \mathrm{wt} / \mathrm{vol})-$ EDTA $(0.02 \% \mathrm{wt} / \mathrm{vol})$ solution (Sigma-Aldrich) for experiments. Immunohistochemical staining of the endothelial monolayer showed strongly positive expression of the endothelial marker, von Willebrand factor. Human recombinant TNF and VEGF were from R\&D Systems and were used at $10 \mathrm{ng} / \mathrm{ml}$. VEGF receptor tyrosine kinase inhibitor SU1498, PI3K inhibitors LY294002 and wortmannin, and PLC- $\gamma$ inhibitor U73122 and its inactive homolog U73343 were purchased from Calbiochem. Transfection of ECs and 293T cells was performed by Lipofectamine 2000 according to the manufacturer's protocol (Gibco). Cells were cultured at $90 \%$ confluence in 6-well plates and were transfected with total $4 \mu \mathrm{g}$ plasmid constructs as indicated. Cells were treated and harvested at 36-48 hours after transfection, and cell lysates were used for protein assays. AIP1 siRNA knockdown was performed as described previously $(18,50)$.

\section{Immunoprecipitation and immunoblotting}

ECs after various treatments were washed twice with cold PBS and harvested in a membrane lysis buffer $(30 \mathrm{mM}$ Tris, $\mathrm{pH} 8.0,10 \mathrm{mM} \mathrm{NaCl}$, $5 \mathrm{mM}$ EDTA, $10 \mathrm{~g} / \mathrm{l}$ polyoxyethylene-8-lauryl ether, $1 \mathrm{mM}$ 0-phenanthroline, $1 \mathrm{mM}$ iodoacetamide, $10 \mathrm{mM} \mathrm{NaF}, 5 \mathrm{mM}$ orthovanadate, and $10 \mathrm{mM}$ sodium pyrophosphate). Cells were immediately frozen in liquid nitrogen. Cell lysates were then thawed on ice, scraped, sonicated, and centrifuged at $14,000 \times g$ at $4^{\circ} \mathrm{C}$ for 15 minutes. Supernatants were used immediately for immunoblot or immunoprecipitation. To analyze protein interaction in vivo via immunoprecipitation, supernatants of cell lysates were diluted 3 times with a cold lysis buffer (50 mM Tris- $\mathrm{HCl}, \mathrm{pH} 7.6,150 \mathrm{mM} \mathrm{NaCl}, 0.1 \%$ Triton X-100, 0.75\% Brij 96, 1 mM sodium orthovanadate, $1 \mathrm{mM}$ sodium fluoride, $1 \mathrm{mM}$ sodium pyrophosphate, $10 \mu \mathrm{g} / \mathrm{ml}$ aprotinin, $10 \mu \mathrm{g} / \mathrm{ml}$ leupeptin, $2 \mathrm{mM}$ PMSF, and $1 \mathrm{mM}$ EDTA). The lysates were then incubated with the first protein-specific antiserum (e.g., anti-VEGFR2) on ice for 1.5 hours. Then, $10 \mu \mathrm{l}$ of protein A/G PLUS-agarose (Santa Cruz Biotechnology Inc.) for was added and incubated for 2 hours with rotation. Immune complexes were collected after each immunoprecipitation by centrifugation at $13,000 \mathrm{~g}$ for 10 minutes followed by $3-5$ washes with lysis buffer. The immune complexes were subjected to SDS-PAGE, followed by immunoblot with the second protein (e.g., phosphotyrosine antibody; Upstate). The chemiluminescence was detected using an ECL kit according to the instructions of the manufacturer (Amersham Life Science). For detection of FLAG-tagged proteins (AIP1), anti-FLAG M2 antibody was used for immunoblot. Antibodies against anti-VEGFR2, phospho-VEGFR2 (pY1054/1059), and AIP1 were described previously $(9,18)$.
Antibodies against phospho-VEGFR2 (pY1175), phospho-Akt (Ser-473), Akt, phospho-PLC- $\gamma$ (pY783) and PLC- $\gamma$, and phospho-JNK and JNK were from Cell Signaling Technology Inc. Anti-FLAG M2 antibody was from Sigma-Aldrich. Protein A/G PLUS-agarose and anti- $\beta$-tubulin were from Santa Cruz Biotechnology Inc.

\section{In vitro EC migration and tube formation assays}

A transwell migration assay. Cell migration was examined using Transwell fitted with polycarbonate filters ( 8 - $\mu \mathrm{m}$ pore size) (Corning Inc.). Briefly, the under surface of the filters were coated overnight at $4{ }^{\circ} \mathrm{C}$ with fibronectin $(20 \mu \mathrm{g} / \mathrm{ml})$ in PBS ( $\mathrm{pH} 7.4)$. The coating solution was removed from the lower chamber before filling with migration medium. The lower chambers were filled with DMEM containing $0.5 \%$ BSA, with or without VEGF (50 ng/ml). Cells were harvested in PBS containing $5 \mathrm{mM}$ EDTA, and after washing with DMEM containing $5 \mathrm{mM} \mathrm{MgCl}_{2}$, cells were resuspended to a concentration of $1 \times 10^{6} \mathrm{cells} / \mathrm{ml}$ DMEM containing $0.5 \%$ BSA. We loaded $1 \times 10^{5}$ cells $(0.1 \mathrm{ml})$ into each upper chamber and cells were cultured for 6 hours at $37^{\circ} \mathrm{C}$ in the presence of $5 \% \mathrm{CO}_{2}$ in a humidified incubator. Cells that did not migrate through the coated filters were removed with cotton swabs, and cells that migrated to the lower surfaces of the filters were stained for 30 minutes with $0.2 \%$ crystal violet in $10 \%$ ethanol. The chambers were washed thoroughly in a water reservoir, and the interior was dried with cotton swabs. Cells on the lower surfaces of the filters were counted under microscope.

Monolayer EC migration. Monolayer EC migration was modified from the method described previously (25). Briefly, infected ECs were cultured in $0.5 \%$ FBS overnight and subjected to "wound injury" assay with a plastic pipette tip (25). Cells were plated with fresh media and were further cultured for 12-24 hours. The EC migration in culture was determined by measuring wound areas in cell monolayers. Three different images from each well along the wound were captured by a digital camera under a microscope $(\times 4)$. Wound area $\left(\mathrm{mm}^{2}\right)$ was measured and analyzed by NIH Image 1.60 (http://rsbweb.nih.gov/nih-image/).

EC tube formation. EC tube formation in Matrigel was performed as follows: 24-well tissue culture plates were coated with $0.2 \mathrm{ml}$ of Matrigel per well and incubated at $37^{\circ} \mathrm{C}$ for 1 hour (Matrigel; BD). HUVECs were serum starved overnight and 2,000 cells were plated on Matrigel $(0.2 \mathrm{ml})$ in a 24 -well plate. At 30 minutes after plating, fresh medium containing the indicated concentrations (see the legend for Figure 6, C and D) of inhibitors in $1 \%$ serum was added and further incubated for 24 hours. A branched EC network was visualized under microscope and photographs were taken using a Leica phase contrast microscope. The area covered by branched cells $\left(\mathrm{mm}^{2}\right)$ was measured and analyzed by NIH Image 1.60 (http://rsbweb.nih.gov/nih-image/).

\section{Statistics}

All data are expressed as mean \pm SEM. Statistical differences were measured by the nonparametric Mann-Whitney $U$ test. A value of $P<0.05$ was considered as statistically significant.

\section{Acknowledgments}

This work was supported by NIH grants R01 HL-65978-5, R01 HL077357-1, and P01HL070295-6, an Established Investigator Award from the American Heart Association (0440172N), and a National Nature Science Foundation of China (30828032) to W. Min, a National Cancer Institute (NCI) Ruth Kirschstein Predoctoral Fellowship Award (F31 CA 136316) to D. Jones, and a Scientific Development Grant from the American Heart Association $(0835544 \mathrm{~N})$ to H. Chen. We thank Jun Yu and Themis Kyriakides for help and discussion. 
Received for publication May 9, 2008, and accepted in revised form September 17, 2008.

Address correspondence to: Wang Min, Interdepartmental Program in Vascular Biology and Therapeutics and Department of Pathology, Yale University School of Medicine, 10 Amistad St., New Haven, Connecticut 06520, USA. Phone: (203) 785-6047;
Fax: (203) 737-2293; E-mail: wang.min@yale.edu. Or to: Hong Chen, Oklahoma Medical Research Foundation, 825 NE 13th St., Oklahoma City, Oklahoma 73104, USA. Phone: (405) 271-2750; Fax: (405) 271-3137; E-mail: hong-chen@omrf.org.

Haifeng Zhang, Yun He, Shengchuan Dai, and Zhe Xu contributed equally to this work.
1. Carmeliet,P. 2003. Angiogenesis in health and disease. Nat. Med. 9:653-660.

2. Ferrara, N., Gerber, H.P., and LeCouter, J. 2003. The biology of VEGF and its receptors. Nat. Med. 9:669-676.

3. Olsson, A.K., Dimberg, A., Kreuger, J., and Claesson-Welsh, L. 2006. VEGF receptor signalling - in control of vascular function. Nat. Rev. Mol. Cell Biol. 7:359-371.

4. Watanabe, K., et al. 2004. Vasohibin as an endothelium-derived negative feedback regulator of angiogenesis. J. Clin. Invest. 114:898-907.

5. Lee, M.J., et al. 1999. Vascular endothelial cell adherens junction assembly and morphogenesis induced by sphingosine-1-phosphate. Cell. 99:301-312.

6. Tanimoto, T., Jin, Z.G., and Berk, B.C. 2002. Transactivation of VEGF receptor Flk- $1 /$ KDR is involved in sphingosine 1-phosphate-stimulated phosphorylation of Akt and eNOS. J. Biol. Chem. 277:42997-43001

7. Autiero, M., et al. 2003. Role of PlGF in the intraand intermolecular cross talk between the VEGF receptors Flt1 and Flk1. Nat. Med. 9:936-943.

8. Jin, Z.G., et al. 2003. Ligand-independent activation of vascular endothelial growth factor receptor 2 by fluid shear stress regulates activation of endothelial nitric oxide synthase. Circ. Res. 93:354-363.

9. Zhang, R., et al. 2003. Etk/Bmx transactivates vascular endothelial growth factor 2 and recruits phosphatidylinositol 3-kinase to mediate the tumor necrosis factor-induced angiogenic pathway. J. Biol. Chem. 278:51267-51276.

10. He, Y., et al. 2006. Critical function of Bmx/Etk in ischemia-mediated arteriogenesis and angiogenesis. J. Clin. Invest. 116:2344-2355.

11. Soker, S., Takashima, S., Miao, H.Q., Neufeld, G., and Klagsbrun, M. 1998. Neuropilin-1 is expressed by endothelial and tumor cells as an isoform-specific receptor for vascular endothelial growth factor. Cell. 92:735-745.

12. Carmeliet, P., et al. 1999. Targeted deficiency or cytosolic truncation of the VE-cadherin gene in mice impairs VEGF-mediated endothelial survival and angiogenesis. Cell. 98:147-157.

13. Soldi, R., et al. 1999. Role of alphavbeta3 integrin in the activation of vascular endothelial growth factor receptor-2. EMBO J. 18:882-892.

14. Weis, S., et al. 2004. Src blockade stabilizes a Flk/ cadherin complex, reducing edema and tissue injury following myocardial infarction. J. Clin. Invest. 113:885-894.

15. Weis, S.M., and Cheresh, D.A. 2005. Pathophysiological consequences of VEGF-induced vascular permeability. Nature. 437:497-504.

16. Tzima, E., et al. 2005. A mechanosensory complex that mediates the endothelial cell response to fluid shear stress. Nature. 437:426-431.

17. Chen, H., Tu, S.W., and Hsieh, J.T. 2005. Downregulation of human DAB2IP gene expression mediated by polycomb Ezh2 complex and histone deacetylase in prostate cancer. J. Biol. Chem. 280:22437-22444.

18. Zhang, R., et al. 2003. AIP1 mediates TNF-alphainduced ASK1 activation by facilitating dissociation of ASK1 from its inhibitor 14-3-3. J. Clin. Invest.
111:1933-1943.

19. Wang, Z., et al. 2002. The mechanism of growthinhibitory effect of DOC-2/DAB2 in prostate cancer. Characterization of a novel GTPase-activating protein associated with $\mathrm{N}$-terminal domain of DOC-2/DAB2. J. Biol. Chem. 277:12622-12631.

20. Dote, H., et al. 2004. Aberrant promoter methylation in human DAB2 interactive protein (hDAB2IP) gene in breast cancer. Clin. Cancer Res. 10:2082-2089.

21. Yano, M., et al. 2005. Aberrant promoter methylation of human DAB2 interactive protein (hDAB2IP) gene in lung cancers. Int. J. Cancer. 113:59-66.

22. Duggan, D., et al. 2007. Two genome-wide association studies of aggressive prostate cancer implicate putative prostate tumor suppressor gene DAB2IP. J. Natl. Cancer Inst. 99:1836-1844.

23. Hsieh, J.T., Karam, J.A., and Min, W. 2007. Genetic and biologic evidence that implicates a gene in aggressive prostate cancer. J. Natl. Cancer Inst. 99:1823-1824.

24. von Bergh, A.R., et al. 2004. Identification of a novel RAS GTPase-activating protein (RASGAP) gene at 9q34 as an MLL fusion partner in a patient with de novo acute myeloid leukemia. Genes Chromosomes Cancer. 39:324-334.

25. Luo, D., et al. 2006. Differential functions of tumor necrosis factor receptor 1 and 2 signaling in ischemia-mediated arteriogenesis and angiogenesis. Am. J. Pathol. 169:1886-1898.

26. Fajardo, L.F., Kowalski, J., Kwan, H.H., Prionas, S.D., and Allison, A.C. 1988. The disc angiogenesis system. Lab. Invest. 58:718-724.

27. Kyriakides, T.R., Zhu, Y.H., Yang, Z., Huynh, G., and Bornstein, P. 2001. Altered extracellular matrix remodeling and angiogenesis in sponge granulomas of thrombospondin 2-null mice. Am. J. Pathol. 159:1255-1262.

28. Heil, M., and Schaper, W. 2004. Influence of mechanical, cellular, and molecular factors on collateral artery growth (arteriogenesis). Circ. Res. 95:449-458.

29. Skoura, A., et al. 2007. Essential role of sphingosine 1 -phosphate receptor 2 in pathological angiogenesis of the mouse retina. J. Clin. Invest. 117:2506-2516.

30. Blezinger, P., et al. 1999. Intratracheal administration of interleukin 12 plasmid-cationic lipid complexes inhibits murine lung metastases. Hum. Gene Ther. 10:723-731.

31. Luo, D., et al. 2008. AIP1 is critical in transducing IRE1-mediated endoplasmic reticulum stress response. J. Biol. Chem. 283:11905-11912.

32. Henkemeyer, M., et al. 1995. Vascular system defects and neuronal apoptosis in mice lacking ras GTPase-activating protein. Nature. 377:695-701.

33. Carmeliet, P., et al. 2001. Synergism between vascular endothelial growth factor and placental growth factor contributes to angiogenesis and plasma extravasation in pathological conditions. Nat. Med. 7:575-583.

34. Aicher, A., et al. 2003. Essential role of endothelial nitric oxide synthase for mobilization of stem and progenitor cells. Nat. Med. 9:1370-1376.

35. Chen, J., et al. 2005. Akt1 regulates pathological angiogenesis, vascular maturation and permeability in vivo. Nat. Med. 11:1188-1196.

36. Yamaoka-Tojo, M., et al. 2004. IQGAP1, a novel vascular endothelial growth factor receptor binding protein, is involved in reactive oxygen species-dependent endothelial migration and proliferation. Circ. Res. 95:276-283.

37. Hart, M.J., Callow, M.G., Souza, B., and Polakis, P. 1996. IQGAP1, a calmodulin-binding protein with a rasGAP-related domain, is a potential effector for cdc42Hs. EMBO J. 15:2997-3005.

38. Mataraza, J.M., Briggs, M.W., Li, Z., Entwistle, A., Ridley, A.J., and Sacks, D.B. 2003. IQGAP1 promotes cell motility and invasion. J. Biol. Chem. 278:41237-41245.

39. Takahashi, T., Yamaguchi, S., Chida, K., and Shibuya, M. 2001. A single autophosphorylation site on KDR/Flk-1 is essential for VEGF-Adependent activation of PLC-gamma and DNA synthesis in vascular endothelial cells. EMBO J. 20:2768-2778.

40. Matsumoto, T., and Claesson-Welsh, L. 2001. VEGF receptor signal transduction. Sci. STKE. 2001:RE21.

41. Duval, M., Bedard-Goulet, S., Delisle, C., and Gratton, J.P. 2003. Vascular endothelial growth factordependent down-regulation of Flk-1/KDR involves Cbl-mediated ubiquitination. Consequences on nitric oxide production from endothelial cells. J. Biol. Chem. 278:20091-20097.

42. Singh, A.J., Meyer, R.D., Band, H., and Rahimi, N. 2005. The carboxyl terminus of VEGFR-2 is required for PKC-mediated down-regulation. Mol. Biol. Cell. 16:2106-2118.

43. Lampugnani, M.G., et al. 2002. VE-cadherin regulates endothelial actin activating Rac and increasing membrane association of Tiam. Mol. Biol. Cell. 13:1175-1189.

44. Huang, L., et al. 1999. НСРТPA, a protein tyrosine phosphatase that regulates vascular endothelial growth factor receptor-mediated signal transduction and biological activity. J. Biol. Chem. 274:38183-38188.

45. Yu, J., et al. 2005. Endothelial nitric oxide synthase is critical for ischemic remodeling, mural cell recruitment, and blood flow reserve. Proc. Natl. Acad. Sci. U. S. A. 102:10999-11004.

46. Kyriakides, T.R., and Bornstein, P. 2003. Matricellular proteins as modulators of wound healing and the foreign body response. Thromb. Haemost. 90:986-992.

47. Miles, A.A., and Miles, E.M. 1952. Vascular reactions to histamine, histamine-liberator and leukotaxine in the skin of guinea-pigs. J. Physiol. 118:228-257.

48. Thurston, G., et al. 1999. Leakage-resistant blood vessels in mice transgenically overexpressing angiopoietin-1. Science. 286:2511-2514.

49. Berenjian, S., and Akusjarvi, G. 2006. Binary AdEasy vector systems designed for Tet-ON or TetOFF regulated control of transgene expression. Virus Res. 115:16-23.

50. Min, W., et al. 2008. AIP1 recruits phosphatase PP2A to ASK1 in tumor necrosis factor-induced ASK1-JNK activation. Circ. Res. 102:840-848. 